\title{
Dynamics of Highly Supercooled Liquids: Heterogeneity, Rheology, and Diffusion
}

\author{
Ryoichi Yamamoto and Akira Onuki \\ Department of Physics, Kyoto University, Kyoto 606-01 \\ E-mail: ryoichi@ton.scphys.kyoto-u.ac.jp, onuki@ton.scphys.kyoto-u.ac.jp
}

(August 27, 2018)

\begin{abstract}
Highly supercooled liquids with soft-core potentials are studied via molecular dynamics simulations in two and three dimensions in quiescent and sheared conditions. We may define bonds between neighboring particle pairs unambiguously owing to the sharpness of the first peak of the pair correlation functions. Upon structural rearrangements, they break collectively in the form of clusters whose sizes grow with lowering the temperature $T$. The bond life time $\tau_{b}$, which depends on $T$ and the shear rate $\dot{\gamma}$, is on the order of the usual structural or $\alpha$ relaxation time $\tau_{\alpha}$ in weak shear $\dot{\gamma} \tau_{\alpha} \ll 1$, while it decreases as $1 / \dot{\gamma}$ in strong shear $\dot{\gamma} \tau_{\alpha} \gg 1$ due to shear-induced cage breakage. Accumulated broken bonds in a time interval $\left(\sim 0.05 \tau_{b}\right)$ closely resemble the critical fluctuations of Ising spin systems. For example, their structure factor is well fitted to the Ornstein-Zernike form, which yields the correlation length $\xi$ representing the maximum size of the clusters composed of broken bonds. We also find a dynamical scaling relation, $\tau_{b} \sim \xi^{z}$, valid for any $T$ and $\dot{\gamma}$ with $z=4$ in two dimensions and $z=2$ in three dimensions. The viscosity is of order $\tau_{b}$ for any $T$ and $\dot{\gamma}$, so marked shear-thinning behavior emerges. The shear stress is close to a limiting stress in a wide shear region. We also examine motion of tagged particles in shear in three dimensions. The diffusion constant is found to be of order $\tau_{b}^{-\nu}$ with $\nu=0.75 \sim 0.8$ for any $T$ and $\dot{\gamma}$, so it is much enhanced in strong shear compared with its value at zero shear. This indicates breakdown of the Einstein-Stokes relation in accord with experiments. Some possible experiments are also proposed.
\end{abstract}

64.70Pf, 83.50Gd, 61.43Fs

\section{INTRODUCTION}

Particle motions in supercooled liquids are severely restricted or jammed, thus giving rise to slow structural relaxations and highly viscoelastic behavior [1,2]. Recently much attention has been paid to the mode coupling theory, 3. 1, which is a first analytic scheme describing onset of slow structural relaxations considerably above $T_{g}$. There, the density fluctuations with wave numbers around the first peak position of the structure factor are of most importance and no long range correlations are predicted. For a long time, however, it has been expected [5] that rearrangements of particle configurations in glassy materials should be cooperative, involving many molecules, owing to configuration restrictions. In other words, such events occur only in the form of clusters whose sizes increase at low temperatures. In normal liquid states, on the contrary, they are frequent and uncorrelated among one another in space and time. Such an idea was first put forth by Adam and Gibbs [5, who invented a frequently used jargon, cooperatively rearranging regions (CRR). However, it is difficult to judge whether or not such phenomenological models are successful in describing real physics and in making quantitative predictions.

Molecular dynamics (MD) simulations can be powerful tools to gain insights into relevant physical processes in highly supercooled liquids. Such processes are often masked in averaged quantities such as the density time correlation functions. As a marked example, we mention kinetic heterogeneities observed in recent simulations [9 18]. Using a simple two dimensional fluid, Muranaka and Hiwatari [9] visualized significant large scale heterogeneities in particle displacements in a relatively short time interval which was supposed to correspond to the $\beta$ relaxation time regime. In liquid states with higher temperatures, Hurley and Harrowell [11] observed similar kinetic heterogeneities but the correlation length was still on the order of a few particle diameters. The characterization of these patterns has not been made in these papers. Recently our simulations on model fluid mixtures in two and three dimensions [13 15$]$ have identified weakly bonded or relatively active regions from breakage of appropriately defined bonds. Spatial distributions of such regions resemble the critical fluctuations in Ising spin systems, so the correlation length $\xi$ can be determined. It grows up to the system size as $T$ is lowered, but no divergence seems to exist at nonzero temperatures [13,19] 21]. Donati et al. have observed string-like clusters whose lengths increase at low temperatures in a three dimensional binary mixture [17]. In addition, Monte Carlo simulations of a dense polymer by Ray and Binder showed a significant system size dependence of the monomer diffusion constant, which indicates heterogeneities over the system size [18].

Most previous papers so far are concerned with nearequilibrium properties such as relaxation of the density time correlation functions or dielectric response. ¿From 
our point of view, these quantities are too restricted or indirect, and there remains a rich group of unexplored problems in far-from-equilibrium states. For example, nonlinear glassy response against electric field, strain, etc. constitutes a future problem 22]. In this paper we apply a simple shear flow $v_{x}=\dot{\gamma} y$ in the $x$ direction and realize steady states 23. The velocity gradient $\dot{\gamma}$ in the $y$ direction is called the shear rate or simply shear. We shall see that it is a relevant perturbation drastically changing the glassy dynamics when $\dot{\gamma}$ exceeds the inverse of the structural or $\alpha$ relaxation time $\tau_{\alpha}$. As is well known, $\tau_{\alpha}$ increases dramatically from microscopic to macroscopic times in a rather narrow temperature range [1,2]. Generally, in near-critical fluids and various complex fluids, nonlinear shear regimes are known to emerge when $\dot{\gamma}$ exceeds some underlying relaxation rate 23. However, in supercooled liquids, it is unique that even very small shear can greatly accelerate the microscopic rearrangement processes. Similar effects are usually expected in systems composed of very large elements such as colloidal suspensions.

Though rheological experiments on glass-forming fluids have not been abundant, Simmons et al found that the viscosity $\eta(\dot{\gamma})=\sigma_{x y} / \dot{\gamma}$ exhibits strong shear-thinning behavior,

$$
\eta(\dot{\gamma}) \cong \eta(0) /\left(1+\dot{\gamma} \tau_{\eta}\right)
$$

in soda-lime-silica glasses in steady states under shear 24 26], $\tau_{\eta}$ being a long rheological time. After application of shear, they also observed overshoots of the shear stress before approach to steady states. Our previous reports 14,15 have treated nonlinear rheology in supercooled liquids in agreement with these these experiments. Interestingly, similar jamming dynamics has begun to be recognized also in rheology of foams 27 29] and granular materials 30] composed of large elements. Shear-thinning behavior and heterogeneities in configuration rearrangements are commonly observed also in these macroscopic systems.

As a closely related problem, understanding of mechanical properties of amorphous metals such as $\mathrm{Cu}_{57} \mathrm{Zr}_{43}$ has been of great technological importance 31 35. They are usually ductile in spite of their high strength. At low temperatures $T \lesssim 0.6 \sim 0.7 T_{g}$ localized bands $(\lesssim 1 \mu \mathrm{m})$, where zonal slip occurs, have been observed above a yield stress. At relatively high temperatures $T \gtrsim 0.6 \sim 0.7 T_{g}$, on the other hand, shear deformations are induced homogeneously (on macroscopic scales) throughout samples, giving rise to viscous flow with strong shear thinning behavior. In particular, in their 3D simulations Takeuchi et al. 34,35 followed atomic motions after application of a small shear strain to observe heterogeneities among poorly and closely packed regions, which are essentially the same entities we have discussed. Our simulations under shear in this paper correspond to the homogeneous regime at relatively high temperatures in amorphous metals.
Another interesting issue is as follows. Several experiments have revealed that the translational diffusion constant $D$ of a tagged particle in a fragile glassy matrix becomes increasingly larger than the Einstein-Stokes value $D_{E S}=k_{B} T / 2 \pi \eta a$ with lowering $T$, where $\eta$ is the (zero-shear) viscosity and $a$ is the diameter of the particle 2.,36 38. In particular, the power law behavior $D \propto \eta^{-\nu}$ with $\nu \cong 0.75$ was observed at sufficiently low temperatures 36]. Thus $D / D_{E S}$ increases from of order 1 up to order $10^{2} \sim 10^{3}$ in supercooling experiments. Furthermore, smaller probe particles exhibit a more pronounced increase of the ratio $D \eta / T \propto D / D_{E S}$ with lowering $T$ [37. It is generally believed that $\eta$ is proportional to the $\alpha$ relaxation time $\tau_{\alpha}$ or the rotational relaxation time $1 / D_{\text {rot }}$ for anisotropic molecules $\left(D_{\text {rot }}\right.$ being the rotational diffusion constant) [36,37,39]. Therefore, individual particles are much more mobile at long times $t \gtrsim \tau_{\alpha}$ than expected from the Stokes-Einstein formula. In a MD simulation on a 3D binary mixture with $N=500$ in 3D 40] the same tendency was apparently seen despite of their small system size. Very recently, in a MD simulation in a 2D binary mixture with $N=1024$, Perera and Harrowell have observed clear deviation from the linear relation $D \propto \tau_{\alpha}$ where $\tau_{\alpha}$ is obtained from the decay of the time correlation function as in our case in Section 6 41]. We will examine this problem in a much larger $3 \mathrm{D}$ system with $N=N_{1}+N_{2}=10^{4}$ generally in the presence of shear, where the viscosity and the diffusion constant both vary tremendously in strong shear $\left(\dot{\gamma} z 1 / \tau_{\alpha}\right)$.

The organization of this paper is as follows. In Sec.II our model binary mixtures and our simulation method will be explained. In Sec.III bonds among particle pairs will be introduced at distances close to the first peak position of the pair correlation functions. Breakage of such bonds will then be followed numerically, which exhibits heterogeneities enhanced at low temperatures. Their analysis will yield the correlation length in Sec. IV. Rheology of supercooled liquids will be studied in Sec.V. These effects were briefly reported in our previous reports 13 15]. In Sec.VI new results on motion of tagged particles will be presented.

\section{MODEL AND SIMULATION METHOD}

We performed MD simulations in two dimensions (2D) and three dimensions (3D) on binary mixtures composed of two different atomic species, 1 and 2, with $N_{1}=N_{2}=$ 5000 particles with the system volume $V$ being fixed. Parameters chosen are mostly common in $2 \mathrm{D}$ and $3 \mathrm{D}$. They interact via the soft-core potential 96 15, 41, 43,

$$
v_{\alpha \beta}(r)=\epsilon\left(\sigma_{\alpha \beta} / r\right)^{12}, \quad \sigma_{\alpha \beta}=\frac{1}{2}\left(\sigma_{\alpha}+\sigma_{\beta}\right),
$$

where $r$ is the distance between two particles and $\alpha, \beta=$ 1,2. The interaction is truncated at $r=4.5 \sigma_{1}$ in $2 \mathrm{D}$ and $r=3 \sigma_{1}$ in 3D. The leapfrog algorithm is used to 
integrate the differential equations with a time step of $0.005 \tau_{0}$, where

$$
\tau_{0}=\left(m_{1} \sigma_{1}^{2} / \epsilon\right)^{1 / 2} .
$$

The space and time are measured in units of $\sigma_{1}$ and $\tau_{0}$. The mass ratio is $m_{2} / m_{1}=2$, while the size ratio is

$$
\sigma_{2} / \sigma_{1}=1.4 \quad(\mathrm{~d}=2), \quad \sigma_{2} / \sigma_{1}=1.2 \quad(\mathrm{~d}=3),
$$

where $d$ is the space dimensionality. This size difference prevents crystallization and produces amorphous states in our systems at low temperatures.

We fixed the particle density at

$$
n=0.8 / \sigma_{1}^{d}
$$

where $n=n_{1}+n_{2}$ is the total number density. The system linear dimension is $L=118$ in $2 \mathrm{D}$ and $L=23.2$ in $3 \mathrm{D}$. Then our systems are highly compressed. In fact, the volume fraction of the particles may be estimated as $\pi\left(\sigma_{1}^{2} n_{1}+\sigma_{2}^{2} n_{2}\right)=0.93$ in $2 \mathrm{D}$ and as $\frac{4}{3} \pi\left(\sigma_{1}^{3} n_{1}+\right.$ $\left.\sigma_{2}^{3} n_{2}\right)=0.57$ in $3 \mathrm{D}$, where overlapped regions are doubly counted. In such cases, according to the Henderson and Leonard theory 43 , 45 , our binary mixtures may be fairly mapped onto one-component fluids with the softcore potential with an effective radius defined by

$$
\sigma_{\text {eff }}^{d}=\sum_{\alpha, \beta=1,2} x_{\alpha} x_{\beta} \sigma_{\alpha \beta}^{d}
$$

where $x_{1}=n_{1} / n$ and $x_{2}=n_{2} / n=1-x_{1}$ are the compositions of the two components and are $1 / 2$ in our case. As in the one component case the thermodynamic state is characterized by a single parameter (effective density),

$$
\Gamma_{\text {eff }}=n\left(\epsilon / k_{B} T\right)^{d / 12} \sigma_{e f f}^{d} .
$$

For example, Bernu et al. [43] confirmed that the equilibrium pressure $p$ may be well fitted to the scaling form, $p / n k_{B} T-1 \cong 6+6.848\left(\Gamma_{e f f}\right)^{4}$, at all $x_{1}$ in $3 \mathrm{D}$. In Tables I and II we list $\Gamma_{\text {eff }}$ chosen in our simulations together with the corresponding scaled temperatures and pressures in $2 \mathrm{D}$ and $3 \mathrm{D}$. Our pressure data excellently agree with the above scaling form for 3D. by

We here introduce the pair correlation functions $g_{\alpha \beta}(r)$

$$
\left\langle\hat{n}_{\alpha}(\boldsymbol{r}) \hat{n}_{\beta}(\mathbf{0})\right\rangle=n_{\alpha} n_{\beta} g_{\alpha \beta}(r)+n_{\alpha} \delta_{\alpha \beta} \delta(\boldsymbol{r}),
$$

where

$$
\hat{n}_{\alpha}(\boldsymbol{r})=\sum_{j} \delta\left(\boldsymbol{r}-\boldsymbol{r}_{\alpha j}\right) \quad(\alpha=1,2)
$$

are the number densities in terms of the particle positions $\boldsymbol{r}_{\alpha j}(\alpha=1,2, j=1, \cdots, N / 2)$. The time dependence is suppressed for simplicity. In a highly compressed state the inter-particle distances between the $\alpha$ and $\beta$ particles are characterized by

$$
\ell_{\alpha \beta}=\sigma_{\alpha \beta}\left(\epsilon / k_{B} T\right)^{1 / 12}
$$

The last factor $\left(\epsilon / k_{B} T\right)^{1 / 12}$ represents the degree of expulsion or penetration from or into the soft-core regions $\left(r<\sigma_{\alpha \beta}\right)$ on particle encounters, though it is not far from 1 in our case. The one-fluid approximation may be justified if the pair correlation functions satisfy

$$
g_{\alpha \beta}(r)=G\left(r / \ell_{\alpha \beta}, \Gamma_{e f f}\right) .
$$

Namely, $g_{\alpha \beta}(r)$ are independent of $\alpha, \beta$, and $x_{1}$ once the distance is scaled by $\ell_{\alpha \beta}$. The pressure is then expressed as 45

$$
\begin{aligned}
\frac{p}{n k_{B} T}-1 & =-\frac{n}{2 d k_{B} T} \sum_{\alpha, \beta} \int d \boldsymbol{r} x_{\alpha} x_{\beta} v_{\alpha \beta}^{\prime}(r) r g_{\alpha \beta}(r) \\
& =6 V_{d} \Gamma_{e f f} \int_{0}^{\infty} d s \frac{1}{s^{13-d}} G\left(s, \Gamma_{e f f}\right)
\end{aligned}
$$

where $v_{\alpha \beta}^{\prime}(r)=d v_{\alpha \beta}(r) / d r$ and $V_{d}$ is the volume of a unit sphere, so it is $4 \pi / 3$ in $3 \mathrm{D}$ and $\pi$ in $2 \mathrm{D}$. We shall see that (2.10) excellently holds around the first peak of the pair correlation functions in our simulations. This fairly supports the one-fluid approximation, because the soft-core potential and the pair correlation functions decrease very abruptly for $r \gtrsim \ell_{\alpha \beta}$ and for $r \lesssim \ell_{\alpha \beta}$, respectively, and the dominant contribution arises from $r \sim \ell_{\alpha \beta} \sim \sigma_{\alpha \beta}$.

In our systems the structural relaxation time becomes very long at low temperatures. Therefore, the annealing time was taken to be at least $10^{5}$ in $2 \mathrm{D}$ and $10^{4}$ in $3 \mathrm{D}$. No appreciable aging effect was detected in the course of taking data in various quantities such as the pressure or the density time correlation function except for the lowest temperature cases, $\Gamma_{\text {eff }}=1.4$ in $2 \mathrm{D}$ and $\Gamma_{\text {eff }}=1.55$ in $3 \mathrm{D}$. A small aging effect remained in the density time correlation function in these exceptional cases, however.

TABLE I. Simulations in 2D.

\begin{tabular}{cccccc}
\hline \hline$\Gamma_{\text {eff }}$ & 1.0 & 1.1 & 1.2 & 1.3 & 1.4 \\
\hline$k_{B} T / \epsilon$ & 2.54 & 1.43 & 0.85 & 0.526 & 0.337 \\
\hline$p / n k_{B} T-1$ & 15.1 & 22.6 & 33.5 & 50.2 & 75.1 \\
\hline \hline
\end{tabular}

TABLE II. Simulations in 3D.

\begin{tabular}{ccccccc}
\hline \hline$\Gamma_{\text {eff }}$ & 1.15 & 1.3 & 1.4 & 1.45 & 1.5 & 1.55 \\
\hline$k_{B} T / \epsilon$ & 0.772 & 0.473 & 0.352 & 0.306 & 0.267 & 0.234 \\
\hline$p / n k_{B} T-1$ & 18.9 & 26.7 & 33.4 & 37.2 & 41.4 & 46.3 \\
\hline \hline
\end{tabular}


Our simulations were performed in the absence and presence of shear flow 46, 47. In the unsheared case $(\dot{\gamma}=0)$ we performed simulations under the microcanonical (constant energy) condition. However, in the sheared case $(\dot{\gamma}>0)$, we kept the temperature at a constant using the Gaussian constraint thermostat to eliminate the viscous heating effect. No difference was detected between the profile-based and profile-unbased thermostats [47, so results with the profile-based thermostat will be presented in this paper. Our method of applying shear is as follows: The system was at rest for $t<0$ for a very long equilibration time and was then sheared for $t>0$. Here we added the average velocity $\dot{\gamma} y$ to the velocities of all the particles in the $x$ direction at $t=0$ and afterwards maintained the shear flow by using the LeeEdwards boundary condition 46,47. Then steady states were realized after a transient time. In our case shear flow serves to destroy glassy structures and produces no long range structure.

\section{PAIR CORRELATIONS AND BOND BREAKAGE}

\section{A. Pair correlations}

Because of the convenience of visualization in $2 \mathrm{D}$, we first present a snapshot of particles at $\Gamma_{\text {eff }}=1.4$ in $2 \mathrm{D}$ in Fig.1, which gives an intuitive picture of the particle configurations. We can see that each particle is touching mostly 6 particles and infrequently 5 particles at distances close to $\sigma_{\alpha \beta}\left(=1.095^{-1} \ell_{\alpha \beta}\right)$. Similar jammed particle configurations can also be found in $3 \mathrm{D}$, where the coordination number of other particles around each particle is about 12 . Then it is natural that the pair correlation functions $g_{\alpha \beta}(r)(\alpha, \beta=1,2)$ have a very sharp peak at $r \cong \sigma_{\alpha \beta}$, as displayed in Fig.2 for $\Gamma_{\text {eff }}=1.4$ in $2 \mathrm{D}$ and $\Gamma_{\text {eff }}=1.55$ in $3 \mathrm{D}$. Furthermore, the heights of these peaks are all close to 7 in $2 \mathrm{D}$ and 4 in 3D. This confirms the scaling form (2.10) around the first peak.

We newly introduce a density variable representing the degree of particle packing by

$$
\hat{\rho}_{e f f}(\boldsymbol{r})=\sigma_{1}^{d} \hat{n}_{1}(\boldsymbol{r})+\sigma_{2}^{d} \hat{n}_{2}(\boldsymbol{r}),
$$

in terms of which the local volume fraction of the softcore regions is $\pi \hat{\rho}_{\text {eff }}(\boldsymbol{r})$ in $2 \mathrm{D}$ and by $(4 \pi / 3) \hat{\rho}_{\text {eff }}(\boldsymbol{r})$ in $3 \mathrm{D}$. We also consider the local composition fluctuation,

$$
\delta \hat{X}(\boldsymbol{r})=\frac{1}{n}\left[x_{2} \hat{n}_{1}(\boldsymbol{r})-x_{1} \hat{n}_{2}(\boldsymbol{r})\right]
$$

where $x_{1}=x_{2}=1 / 2$ in our case. In Fig.3 we show the corresponding, dimensionless structure factors,

$$
\begin{gathered}
S_{\rho \rho}(q)=\sigma_{1}^{-d} \int d \boldsymbol{r} e^{i \boldsymbol{q} \cdot \boldsymbol{r}}\left\langle\delta \hat{\rho}_{e f f}(\boldsymbol{r}) \delta \hat{\rho}_{e f f}(\mathbf{0})\right\rangle \\
S_{\rho X}(q)=\sigma_{1}^{-d} \int d \boldsymbol{r} e^{i \boldsymbol{q} \cdot \boldsymbol{r}}\left\langle\delta \hat{\rho}_{e f f}(\boldsymbol{r}) \delta \hat{X}(\mathbf{0})\right\rangle
\end{gathered}
$$

$$
S_{X X}(q)=\sigma_{1}^{-d} \int d \boldsymbol{r} e^{i \boldsymbol{q} \cdot \boldsymbol{r}}\langle\delta \hat{X}(\boldsymbol{r}) \delta \hat{X}(\mathbf{0})\rangle
$$

where $\delta \hat{\rho}_{\text {eff }}=\hat{\rho}_{\text {eff }}-\left\langle\hat{\rho}_{\text {eff }}\right\rangle$. They are linear combinations of the usual structure factors,

$$
S_{\alpha \beta}(q)=n_{\alpha} n_{\beta} \int d \boldsymbol{r} e^{i \boldsymbol{q} \cdot \boldsymbol{r}}\left[g_{\alpha \beta}(r)-1\right]
$$

from the definitions (3.1) and (3.2). The temperatures in Fig. 3 are common to those in Fig.2. Note that the dimensionless wavenumber $q$ is measured in units of $\sigma_{1}^{-1}$. The $S_{\rho \rho}(q)$ has a pronounced peak at $q \sim 6$ and becomes very small $(\sim 0.01)$ at smaller $q$ both in $2 \mathrm{D}$ and $3 \mathrm{D}$. In this sense our systems are highly incompressible at long wavelengths. On the other hand, $S_{X X}(q)$ has no peak and is roughly a constant over a very wide $q$ region, suggesting no enhancement of the composition fluctuations and no tendency of phase separation at least in our simulation times.

¿From Fig.3 we may estimate the magnitude of the isothermal compressibility $K_{T X}=(\partial n / \partial p)_{T X} / n$. In equilibrium it is expressed in terms of the fluctuation variances as

$$
\begin{aligned}
& k_{B} T K_{T X}=n^{-4} \lim _{q \rightarrow 0}\left[S_{11}(q) S_{22}(q)-S_{12}(q)^{2}\right] / S_{X X}(q) \\
& =\frac{\sigma_{1}^{d}}{\left(\sigma_{1}^{d} n_{1}+\sigma_{2}^{d} n_{2}\right)^{2}} \lim _{q \rightarrow 0}\left[S_{\rho \rho}(q)-S_{\rho X}(q)^{2} / S_{X X}(q)\right] .
\end{aligned}
$$

The first line was the expression in Ref. 48, and the second line follows if use is made of (3.1) and (3.2). The dimensionless combination $n k_{B} T K_{T X}$ is equal to 0.0028 in $2 \mathrm{D}$ and 0.0067 in $3 \mathrm{D}$. If we assume that the adiabatic compressibility $K_{s X}=(\partial n / \partial p)_{s X} / n$ is of the same order as $K_{T X}$, the sound speed $c$ turns out to be of order 10 in units of $\sigma_{1} / \tau_{0}$.

Our structure factors were obtained by time averaging over very long times, which are $10^{5}$ for $2 \mathrm{D}$ and $10^{4}$ for 3D. However, irregular shapes of $S_{X X}(q)$ persisted at long wavelengths $q \lesssim 1$. Such large scale composition fluctuations have very long life times $\left(\gg \tau_{\alpha}\right)$ and are virtually frozen throughout the simulation. Therefore, we admit the possibility that our supercooled states at low temperatures might phase-separate to form crystalline regions on much longer time scales. On the contrary, the long wavelength fluctuations of $\hat{\rho}_{\text {eff }}$ have much shorter time scales, probably they vary on acoustic time scales $\sim 1 / c q$.

As is well known, the temperature dependence of the static pair correlation functions is much milder than that of the dynamical quantities. Similarly, their shear dependence is also mild even for $\dot{\gamma} \tau_{\alpha} \gg 1$ as long as $\dot{\gamma} \ll 1$. In particular, their spatially anisotropic part is at most a few percents of their isotropic part around the first peak 
positions $r \cong \sigma_{\alpha \beta}$ in our case. This is consistent with the fact that the attained shear stress in our simulations are at most a few percents of the particularly high pressure $p$ of our systems. Note that the average shear stress $\sigma_{x y}$ in sheared steady states may be related to the steady state pair correlation functions $g_{\alpha \beta}(\boldsymbol{r})$ as 45]

$$
\sigma_{x y}=-\frac{1}{2} \sum_{\alpha, \beta} n_{\alpha} n_{\beta} \int d \boldsymbol{r} v_{\alpha \beta}^{\prime}(r) \frac{r_{x} r_{y}}{r} g_{\alpha \beta}(\boldsymbol{r}),
$$

where $r_{x}$ and $r_{y}$ are the $x$ and $y$ components of the vector $\boldsymbol{r}$ connecting particle pairs. The dominant contribution here arises from the anisotropy at $r \cong \sigma_{\alpha \beta}$.

\section{B. Bond breakage}

Because of the sharpness of the first peak of $g_{\alpha \beta}(\boldsymbol{r})$ in our systems, we can unambiguously define bonds between particle pairs at distances close to $\sigma_{\alpha \beta}$ in the absence and presence of shear. Such bonds will be broken on the structural $(\alpha)$ relaxation time, because the bond breakage takes place on local configurational rearrangements. We define the bonds as follows. For each atomic configuration given at time $t_{0}$, a pair of particles $i$ and $j$ is considered to be bonded if

$$
r_{i j}\left(t_{0}\right)=\left|\mathbf{r}_{i}\left(t_{0}\right)-\mathbf{r}_{j}\left(t_{0}\right)\right| \leq A_{1} \sigma_{\alpha \beta}
$$

where $i$ and $j$ belong to the species $\alpha$ and $\beta$, respectively. We have set $A_{1}=1.1$ for $2 \mathrm{D}$ and 1.5 for $3 \mathrm{D}$. The resultant bond numbers between $\alpha$ and $\beta$ pairs, $N_{b \alpha \beta}$, are related to the first peak structure of $g_{\alpha \beta}(r)$ as follows. We consider the coordination number $\nu_{\alpha \beta}$ of $\beta$ particles around a $\alpha$ particle within the distance $A_{1} \sigma_{\alpha \beta}$ [43],

$$
\nu_{\alpha \beta}=n_{\beta} \int_{r<A_{1} \sigma_{\alpha \beta}} d \boldsymbol{r} g_{\alpha \beta}(r) \sim C n_{\beta} \sigma_{\alpha \beta}^{d},
$$

where $C$ is about 5 in $2 \mathrm{D}$ and 12 in $3 \mathrm{D}$. Then we simply have

$N_{b \alpha \alpha}=\frac{1}{2} N_{\alpha} \nu_{\alpha \alpha} \quad(\alpha=1,2), \quad N_{b 12}=\frac{1}{2} N_{1} \nu_{12}+\frac{1}{2} N_{2} \nu_{21}$.

In $2 \mathrm{D}$ at $\Gamma_{\text {eff }}=1.4$, we find $\nu_{11}=2.19, \nu_{12}=\nu_{21}=2.54$, and $\nu_{22}=3.41$, which are consistent with the bond numbers, $N_{b 11}=5514, N_{b 11}=13135$, and $N_{b 22}=8436$, counted in a simulation. In $3 \mathrm{D}$ at $\Gamma_{\text {eff }}=1.55$, these numbers are $\nu_{11}=5.57, \nu_{12}=\nu_{21}=6.90, \nu_{22}=8.30$, which are again consistent with $N_{b 11}=13925, N_{b 11}=$ 34476 , and $N_{b 22}=20744$ in a simulation. We stress that our bond definition is insensitive to $A_{1}$, owing to the sharpness of the first peak, as long as it is somewhat larger than 1 and smaller than the second peak distances divided by $\sigma_{\alpha \beta}$.

After a lapse of time $\Delta t$, a pair is regarded to have been broken if

$$
r_{i j}\left(t_{0}+\Delta t\right)>A_{2} \sigma_{\alpha \beta}
$$

with $A_{2}=1.6$ for $2 \mathrm{D}$ and 1.5 for $3 \mathrm{D}$. This definition of bond breakage is also insensitive to $A_{2}$ as long as $A_{2} \geq A_{1}$ and $A_{2} \sigma_{\alpha \beta}$ is shorter than the second peak position of $g_{\alpha \beta}(r)$. We have followed the relaxation of the total surviving (unbroken) bonds $N_{\text {bond }}(\Delta t)$ from the initial number

$$
N_{\text {bond }}(0)=N_{b 11}+N_{b 12}+N_{b 22}
$$

to zero with increasing $\Delta t$. No significant difference has been found between the bond breakage processes of the three kinds of bonds, 1-1, 1-2, and 2-2, so we consider their sum only. We define the bond breakage time $\tau_{b}$ by

$$
N_{\text {bond }}\left(\tau_{b}\right)=N_{\text {bond }}(0) / e .
$$

The relaxation is not simply exponential at low temperatures, apparently because of large scale heterogeneities composed of relatively weakly and strongly bonded regions. If we fit $N_{\text {bond }}(\Delta t)$ to the stretched exponential form, $N_{\text {bond }}(\Delta t) \sim \exp \left[-\left(\Delta t / \tau_{b}\right)^{a^{\prime}}\right]$, the exponent $a^{\prime}$ is close to 1 at relatively high temperatures but is considerably smaller than 1 at the lowest temperatures (for example, $a^{\prime} \sim 0.6$ at $\Gamma_{\text {eff }}=1.55$ in $\left.3 \mathrm{D}\right)$.

In Fig. 4 we show the bond breakage time $\tau_{b}=\tau_{b}(T)$ in the absence of shear as a function of the temperature. It grows strongly with decreasing the temperature. As will be shown in (6.8) in Sec. III, the bond breakage time $\tau_{b}$ is proportional to the $\alpha$ relaxation time $\tau_{\alpha}$ obtained from the decay of the self part of the time correlation function $F_{s}(q, t)$ at $q=2 \pi$. The shear dependence of the bond breakage time $\tau_{b}=\tau_{b}(\dot{\gamma})$ is also of great interest. As shown in Fig. 5 , the bond breakage rate $1 / \tau_{b}(\dot{\gamma})$ consists of the thermal breakage rate $1 / \tau_{b}(0)$ strongly dependent on $T$ and a shear-induced breakage rate proportional to $\dot{\gamma}$. It is expressed in the simplest conceivable form,

$$
1 / \tau_{b}(\dot{\gamma}) \cong 1 / \tau_{b}(0)+A_{b} \dot{\gamma}
$$

where $A_{b}=0.57$ in $2 \mathrm{D}$ and 0.80 in $3 \mathrm{D}$. In the strong shear condition $\dot{\gamma} \tau_{b}(0)>1$, jump motions are induced by shear on the time scale of $1 / \dot{\gamma}$. We shall see that the bond breakage occurs more homogeneously with increasing shear. Therefore, it is natural that, when the strain $\gamma=\dot{\gamma} \Delta t$ reaches 1 , a large fraction of bonds have been broken by shear.

\section{HETEROGENEITY IN BOND BREAKAGE}

Following the bond breakage process we can visualize the kinetic heterogeneity without ambiguity and quantitatively characterize the heterogeneous patterns. In Fig.6 we show spatial distributions of broken bonds in a time interval of $\left[t_{0}, t_{0}+0.05 \tau_{b}\right]$ in $2 \mathrm{D}$, where about $5 \%$ of the initial bonds defined at $t=t_{0}$ have been broken. The 
dots are the center positions $\mathbf{R}_{i j}=\frac{1}{2}\left(\boldsymbol{r}_{i}\left(t_{0}\right)+\boldsymbol{r}_{j}\left(t_{0}\right)\right)$ of the broken pairs at the initial time $t_{0}$. The broken bonds are seen to form clusters with various sizes. The heterogeneity is marked in the glassy case (b) with $\Gamma_{\text {eff }}=1.4$ and $\dot{\gamma}=0$, whereas it is much weaker for the liquid case (a) with $\Gamma_{\text {eff }}=1$ and $\dot{\gamma}=0$. The bond breakage time $\tau_{b}$ is 17 in (a) and $5 \times 10^{4}$ in (b). In (c) we set $\dot{\gamma}=0.25 \times 10^{-2}$ and $\Gamma_{\text {eff }}=1.4$ with $\tau_{b}=32 \sim 1 / \dot{\gamma}$. The heterogeneity is known to become much suppressed by shear, while its spatial anisotropy remains small. Notice that even in normal liquids bond breakage events frequently occur in the form of strings involving a few to several particles, obviously because of the high density of our system. In glassy states such strings become longer and aggregate forming large scale clusters. In 3D we also observe string-like jump motions in accord with Ref. [17] and aggregation of such strings at low temperatures.

In Fig.7 we write the broken bonds in two consecutive time intervals, $\left[t_{0}, t_{0}+0.05 \tau_{b}\right]$ and $\left[t_{0}+0.05 \tau_{b}, t_{0}+0.1 \tau_{b}\right]$ at $\Gamma_{\text {eff }}=1.4$ and $\dot{\gamma}=0$. The clusters of broken bonds in the two time intervals mostly overlap or are adjacent to one another. This demonstrates that weakly bonded regions or collectively rearranging regions ( $\mathrm{CRR}$ ) follow complex space-time evolution on the scales of $\xi$ and $\tau_{b}$. We do not know its evolution laws but will encounter a dynamical scaling law between $\xi$ and $\tau_{b}$ in (4.4) below.

We define the structure factor $S_{b}(q)$ of the broken bonds as

$$
S_{b}(q)=\frac{1}{N_{b}}\left\langle\left|\sum_{<i, j>} \exp \left(i \mathbf{q} \cdot \mathbf{R}_{i j}\right)\right|^{2}\right\rangle
$$

where the summation is over the broken pairs, $N_{b}$ is the total number of the broken bonds in a time interval $\left[t_{0}, t_{0}+\Delta t\right]$, and the angular average over the direction of the wave vector has been taken. Furthermore, we have averaged over 5-50 $S_{b}(q)$ data calculated from sequential configurations of broken bonds. Fig.8 displays the resultant $S_{b}(q)$ after these averaging procedures on logarithmic scales at several $\Gamma_{\text {eff }}$ without shear. The enhancement of $S_{b}(q)$ at small $q$ arises from large scale kinetic heterogeneities growing with increasing $\Gamma_{\text {eff }}$ both in $2 \mathrm{D}$ and $3 \mathrm{D}$. ¿From a plot of $1 / S_{b}(q)$ versus $q^{2}$ in our previous reports [13], we already found that $S_{b}(q)$ can be nicely fitted to the Ornstein-Zernike (OZ) form :

$$
S_{b}(q)=S_{b}(0) /\left(1+\xi^{2} q^{2}\right)
$$

The correlation length $\xi$ is determined from this expression. It grows up to the system length at the lowest temperatures and is insensitive to the width of the time interval $\Delta t$ as long as it is considerably shorter than the bond breakage time $\tau_{b}$ [13]. The agreement of our $S_{b}(q)$ with the OZ form becomes more evident in the plots of $S_{b}(q) / S_{b}(0)$ versus $q \xi$ in Fig.9, in which all the data collapse onto a single OZ master curve both in $2 \mathrm{D}$ and $3 \mathrm{D}$. In particular, in 3D the deviations are very small, although $\xi \sim L$ for low $T$ and small $\dot{\gamma}$ in our case.
We also notice that $S_{b}(q)$ is insensitive to the temperature at large $q$, so from the OZ form (4.2) we find

$$
S_{b}(0) \sim \xi^{2}
$$

The clusters of the broken bonds are thus very analogous to the the critical fluctuations in Ising spin systems. In fact, small scale heterogeneities with sizes $\ell$ in the region $1 \ll \ell \ll \xi$ are insensitive to the temperature. The relation (4.3) is analogous to the relation, $\chi \propto \xi^{2-\eta}$, in Ising spin systems between the magnetic susceptibility $\chi=\lim _{q \rightarrow 0} S(q)$ and the correlation length $\xi$ near the critical point. Here $S(q)$ is the spin structure factor and $\eta$ is the Fisher critical exponent ( $\ll 1$ in $3 \mathrm{D})$.

Obviously, $\xi$ represents the order of the maximum length of the clusters. However, Adam and Gibbs [5] intuitively expected that the minimum size of CRR increases as $\exp \left(\right.$ const. $\left./\left(T-T_{0}\right)\right)$ on lowering $T$ towards $T_{0}$. It has also been discussed as to whether or not there is an underlying thermodynamic phase transition at a nonzero temperature $T_{0}$ in highly supercooled liquids 119 21. ¿From our data we cannot detect any divergence of $\xi$ at a nonzero temperature, although this is not conclusive due to the finite size effect arising from $\xi \sim L$.

Furthermore, as in critical dynamics, we have confirmed a dynamical scaling relation between the bond breakage time $\tau_{b}$ and the correlation length $\xi$,

$$
\tau_{b} \cong A \xi^{z}
$$

where $z=4$ in 2D 41] and $z=2$ in 3D. The coefficient $A$ is independent of $\Gamma_{\text {eff }}$ and $\dot{\gamma}$ chosen in our simulations, as shown in Fig.10 (a) and (b). Notice that the data points at the largest $\xi$ in Fig.10 are those at zero shear for each $\Gamma_{\text {eff }}$. At present we cannot explain the origin of these simple numbers for $z$. We may only argue that $z$ should be larger in 2D than in 3D because of stronger configurational restrictions in $2 \mathrm{D}$. It is surprising that (4.4) holds even in strong shear $\dot{\gamma} \tau_{b}(0) \gg 1$, where the correlation length is independent of $T$ and is determined by shear as

$$
\xi \sim \dot{\gamma}^{-1 / z}
$$

In Fig.10b for 3D, however, we notice $\xi>L$ at $\Gamma_{\text {eff }}=$ 1.50 and 1.55 for weak shear. At present we cannot assess influence of this finite size effect.

In a zeroth order approximation, therefore, the kinetic heterogeneities are characterized by a single parameter, $\xi$ or $\tau_{b}$, owing to the small space anisotropy induced by shear in our systems. The shear rate $\dot{\gamma}$ is apparently playing a role similar to a magnetic field $h$ in Ising spin systems. Thus, $\dot{\gamma}$ and $T$ are two relevant external parameters in supercooled liquids, while $h$ and the reduced temperature $\left(T-T_{c}\right) / T_{c}$ are two relevant scaling fields in Ising systems. 


\section{SUPERCOOLED LIQUID RHEOLOGY}

We next examine nonlinear rheology in our fluid mixtures in supercooled amorphous states. We first display in Fig.11 the shear-dependent viscosity $\eta(\dot{\gamma})$ (in units of $\left.\epsilon \tau_{0} / \sigma_{1}^{d}\right)$ versus $\dot{\gamma}$ in steady states at various $\Gamma_{\text {eff }}$ in $2 \mathrm{D}$ and $3 \mathrm{D}$. This rheological behavior is similar to those in the experiments [24 26]. The viscosity is much enhanced at large $\Gamma_{\text {eff }}$ (low $T$ ) and at low shear, but it tends to be independent of $T$ at very high shear. Remarkably, glassy states exhibit large non-Newtonian behavior even when $\dot{\gamma}$ is much smaller than the microscopic frequency $1 / \tau_{0}=1$, whereas such large effects are expected to appear only for $\dot{\gamma} \sim 1 / \tau_{0}$ in normal liquids far from the critical point 47,49 .

In Fig.12 we demonstrate that the viscosity $\eta(\dot{\gamma})=$ $\sigma_{\alpha \beta} / \dot{\gamma}$ is determined solely by the bond breakage time $\tau_{b}(\dot{\gamma})$ in $(3.15)$ as

$$
\eta(\dot{\gamma}) \cong A_{\eta} \tau_{b}(\dot{\gamma})+\eta_{B},
$$

where $A_{\eta}$ and $\eta_{B}$ are 0.34 and 6.25 in $2 \mathrm{D}$, and 0.24 and 2.2 in $3 \mathrm{D}$, respectively. Because the linearity $\eta \propto \tau_{b}$ is systematically violated at small $\tau_{b}$, the presence of the background viscosity $\eta_{B}$ independent of $\Gamma_{\text {eff }}$ and $\dot{\gamma}$ may be concluded. Note that the effective exponent $(\dot{\gamma} / \eta)(d \eta / d \dot{\gamma})$ remains about -0.8 in Fig.11. As well as the kinetic heterogeneities, steady state rheology is determined only by a single parameter, $\tau_{b}$ or $\xi$. This suggests that a sheared steady state can be fairly mapped onto a quiescent state with a higher temperature but with the same $\xi$.

Substitution of (3.15) then yields

$$
\eta(\dot{\gamma}) \cong A_{\eta} /\left[\tau_{b}(0)^{-1}+A_{b} \dot{\gamma}\right]+\eta_{B},
$$

This form coincides with the empirical law (1.1) by Simons et al. [24,25]. Fig.13 shows that the ratio $\left(\eta(\dot{\gamma})-\eta_{B}\right) /\left(\eta(0)-\eta_{B}\right)$ can be fitted to the universal curve $1 /\left(1+A_{b} x\right)$ with $x=\dot{\gamma} \tau_{b}(0)$ independently of $\Gamma_{\text {eff }}$ both in $2 \mathrm{D}$ and $3 \mathrm{D}$. In strong shear $\dot{\gamma} \tau_{b}(0) \gg 1$, we have the temperature-independent behavior $\eta(\dot{\gamma}) \cong$ $\left(A_{\eta} / A_{b}\right) / \dot{\gamma}+\eta_{B}$, which is evidently seen in Fig.11. If the background viscosity is negligible, a constant limiting stress follows as

$$
\sigma_{x y} \cong \sigma_{\text {lim }}=A_{\eta} / A_{b},
$$

which holds for

$$
1 / \tau_{b}(0) \ll \dot{\gamma} \ll \sigma_{\min } / \eta_{B} \sim 0.1 / \tau_{0} .
$$

Here $\sigma_{\text {lim }}$ is 0.59 in $2 \mathrm{D}$ and 0.30 in 3D in units of $\epsilon / \sigma_{1}^{d}$ and is typically a few percents of the pressure in our systems. The upper bound in (5.4) is very large in usual glass-forming liquids but should be attainable in colloidal systems, while the lower bound can be very small with lowering $T$.
We will argue to derive the above behavior intuitively. Supercooled liquids behave as solids against infinitesimal strain on time scales shorter than $\tau_{b}(0)$ even if the temperature is considerably above the so-called glass transition temperature. Fluid-like behavior is realized only after the bond breakage processes. It is natural that the viscosity is of order $\tau_{b}(0)$ in the linear regime. This is usually justified from the time correlation function expression for the viscosity in terms of the $x y$ component of the stress tensor 445. In strong shear, on the other hand, the bond breakage occurs on the time scale of $1 / \dot{\gamma}$. Upon each bond breakage induced by shear, the particles involved release a potential energy $\epsilon_{r}$ whose maximum is $\epsilon$. There should be a distribution of $\epsilon_{r}$, but let us assume $\epsilon_{r} \sim \epsilon$ for simplicity. It is then instantaneously changed into energies of random motions (and probably sounds) supported by the surrounding particles. The heat transport is rapid in this dissipative process. Because of this and also because of the background thermal motions superposed, we have not detected clear temperature inhomogeneities like hot spots around broken bonds in our simulations. The heat production rate is estimated as

$$
Q \sim n \epsilon / \tau_{b}(\dot{\gamma}) \sim n \epsilon \dot{\gamma},
$$

where $n$ is the number density. Because $Q$ is related to the viscosity by $Q=\sigma_{x y} \dot{\gamma}=\eta(\dot{\gamma}) \dot{\gamma}^{2}$, we obtain

$$
\sigma_{x y}=\eta(\dot{\gamma}) \dot{\gamma} \sim n \epsilon,
$$

in high shear, so $\sigma_{l i m} \sim n \epsilon$. Due to disordered particle configurations, however, it is natural to consider a distribution of the released energy $\epsilon_{r}$, which will explain the viscosity behavior at lower shear. Such a distribution was calculated for a model foam system in shear flow by Durian [28].

\section{MOTION OF TAGGED PARTICLES}

In this section we will follow the motion of tagged particles in a glassy matrix both in the absence and presence of shear in 3D. We will present results only in three dimensions. We first plot in Fig.14 the self part of the density time correlation function for various $\Gamma_{e f f}$ in the usual zero shear condition,

$$
F_{s}(q, t)=\frac{1}{N_{1}}\left\langle\sum_{j=1}^{N_{1}} \exp \left[i \boldsymbol{q} \cdot \Delta \boldsymbol{r}_{j}(t)\right]\right\rangle,
$$

where $q=2 \pi, \Delta \boldsymbol{r}_{j}(t)=\boldsymbol{r}_{j}(t)-\boldsymbol{r}_{j}(0)$, and the summation is taken over all the particles of the species 1 . This function is proportional to the (incoherent) scattering amplitude from labelled particles. As is well known, this function has a plateau at low temperatures $\left(\Gamma_{e f f} \gtrsim 1.45\right.$ in our case), during which the particle is trapped in a cage. After a long time the cage eventually breaks, resulting in diffusion with a very small diffusion constant 
$D$. In this paper we define the $\alpha$ relaxation time $\tau_{\alpha}$ such that $F_{s}\left(q, \tau_{\alpha}\right)=e^{-1}$ at $q=2 \pi$.

We generalize the time correlation function (6.1) in the presence of shear flow by introducing a new displacement vector of the $j$-th particle as

$$
\Delta \boldsymbol{r}_{j}(t)=\boldsymbol{r}_{j}(t)-\dot{\gamma} \int_{0}^{t} d t^{\prime} y_{j}\left(t^{\prime}\right) \boldsymbol{e}_{x}-\boldsymbol{r}_{j}(0),
$$

where $\boldsymbol{e}_{x}$ is the unit vector in the $x$ (flow) direction. In this displacement, the contribution from convective transport by the average flow has been subtracted, which can be known from the time derivative,

$$
\frac{\partial}{\partial t} \Delta \boldsymbol{r}_{j}(t)=\boldsymbol{v}_{j}(t)-\dot{\gamma} y_{j}(t) \boldsymbol{e}_{x}
$$

To get clear understanding of the meaning of this subtraction, let us consider a Brownian particle placed in shear flow as a simple example. On time scales longer than the relaxation time of its velocity, its position $\boldsymbol{r}(t)$ obeys

$$
\frac{\partial}{\partial t} \boldsymbol{r}(t)=\dot{\gamma} y(t) \boldsymbol{e}_{x}+\boldsymbol{f}(t)
$$

where $\boldsymbol{f}(t)$ is the Gaussian random force characterized by $\left\langle f_{\mu}(t) f_{\nu}\left(t^{\prime}\right)\right\rangle=2 D \delta_{\mu \nu} \delta\left(t-t^{\prime}\right)(\mu, \nu=x, y, z)$. Then the modified displacement vector reads

$$
\Delta \boldsymbol{r}(t) \equiv \boldsymbol{r}(t)-\dot{\gamma} \int_{0}^{t} d t^{\prime} y\left(t^{\prime}\right) \boldsymbol{e}_{x}-\boldsymbol{r}(0)=\int_{0}^{t} d t^{\prime} \mathbf{f}\left(t^{\prime}\right)
$$

Here the convective effect does not appear explicitly and the diffusion behavior follows as

$$
\left\langle\Delta \boldsymbol{r}(t)^{2}\right\rangle=6 D t
$$

On the other hand, in the incoherent scattering amplitude, $\Delta \boldsymbol{r}_{j}(t)$ in (6.1) should be taken as the net displacement $\boldsymbol{r}_{j}(t)-\boldsymbol{r}_{j}(0)$ even in shear flow. If $q_{x} \neq 0$, it strongly depends on the thickness of the scattering region in the $y$ (velocity gradient) direction due to a positiondependent Doppler effect [23,50]. Only for $q_{x}=0$, it is proportional to $F_{s}(q, t)$ in the above definition.

Fig. 15 shows $F_{s}(q, t)$ at $q=2 \pi$ for various $\dot{\gamma}$ with a fixed temperature, $\Gamma_{\text {eff }}=1.5$ or $k_{B} T / \epsilon=0.267$ in 3D. Comparison of this figure with Fig.14 suggests that applying shear is equivalent to raising the temperature. Recall that we have made the same statement in analyzing the bond structure factor $S_{b}(q)$ and the nonlinear rheology. Also we may define the shear dependent $\alpha$ relaxation time $\tau_{\alpha}=\tau_{\alpha}(\dot{\gamma})$ by

$$
F_{s}\left(q, \tau_{\alpha}\right)=e^{-1}
$$

In Fig. 16 we recognize that $\tau_{\alpha}$ is proportional to the bond life time $\tau_{b}$ as

$$
\tau_{\alpha} \cong 0.1 \tau_{b}
$$

This relation holds for any $\Gamma_{\text {eff }}$ and $\dot{\gamma}$ in our 3D simulations. The decay of $F_{s}(q, t)$ is not exponential for large $\tau_{\alpha}$. If it is fitted to the stretched exponential form $\exp \left[-\left(t / \tau_{\alpha}\right)^{a}\right]$ around $t \sim \tau_{\alpha}$, the exponent $a$ is increased from values about 0.8 to 1 with increasing $\dot{\gamma}$ as well as with raising $T$. Furthermore, the time correlation function (6.1) has turned out to be almost independent of the direction of the wave vector $\boldsymbol{q}$.

Next it is convenient to analyze the mean square displacement of tagged particles of the species 1 ,

$$
\left\langle(\Delta \boldsymbol{r}(t))^{2}\right\rangle=\frac{1}{N_{1}} \sum_{j=1}^{N_{1}}\left\langle\left(\Delta \boldsymbol{r}_{j}(t)\right)^{2}\right\rangle
$$

Fig.17 shows the transition from the ballistic behavior $\left\langle(\Delta \boldsymbol{r}(t))^{2}\right\rangle \cong 3\left(k_{B} T / m_{1}\right) t^{2}$ to the diffusion behavior $\left\langle(\Delta \boldsymbol{r}(t))^{2}\right\rangle \cong 6 D t$ in shear flow at $\Gamma_{\text {eff }}=1.55$. The arrows in the figure indicate the $\alpha$ relaxation time $\tau_{\alpha}(\dot{\gamma})$. The crossover occurs around $t \sim \tau_{\alpha}$. Fig.18 demonstrates the surprising isotropy of the statistical distribution of $\Delta \boldsymbol{r}_{i}(t)$, where the mean square displacements of the $x, y$, and $z$ components of the vector $\Delta \boldsymbol{r}_{j}(t)$ are separately displayed. We can thus determine $D$ from the mean square displacement in addition to $\tau_{\alpha}$ in shear flow. Note that the $x$ component in Fig.18 is not the usual mean square displacement due to the second term in (6.2). In the appendix we will consider the variances of the net displacement vector $\boldsymbol{r}_{j}(t)-\boldsymbol{r}_{j}(0)$.

Fig.19 shows the shear rate dependences of the viscosity $\eta\left(\sim \tau_{\alpha}\right)$ and the inverse diffusion constant $D^{-1}$ from the linear $\left(\dot{\gamma} \lesssim 10^{-5}\right)$ to the non-Newtonian regime at $\Gamma_{\text {eff }}=1.55$ in $3 \mathrm{D}$, where $D$ is measured in units of $\sigma_{1}^{2} / \tau_{0}$ and $\eta$ in units of $\epsilon \tau_{0} / \sigma_{1}^{d}$. We deduce the relation $D^{-1} \sim \dot{\gamma}^{-\nu}$ with $\nu=0.75 \sim 0.80$ in agreement of the experiment 36], which is appreciably milder than the viscosity decrease $\eta \sim \tau_{\alpha} \sim \dot{\gamma}^{-1}$. In Fig.20 we plot $D$ versus $\eta / k_{B} T$ (in units of $\tau_{0} / \sigma_{1}^{d}$ ) obtained for various $\Gamma_{\text {eff }}$ and $\dot{\gamma}$. The Einstein-Stokes formula, which holds excellently in normal liquids, appears to be violated in supercooled liquids as the other simulations have suggested 40,41]. It is widely believed that this breakdown is a natural consequence of the dynamic heterogeneity in glassy states [36 38]. Detailed numerical analysis will appear in a forthcoming paper.

In our case $\eta / k_{B} T$ changes over 4 decades until $\xi$ reaches the system dimension $L$, whereas it has been changed over 12 decades in the experiments 36, 37. Though the same tendency indicating the breakdown of the Einstein-Stokes relation has been obtained in our simulation, we should admit that our system size in $3 \mathrm{D}$ is not yet sufficiently large and our data at $\Gamma_{\text {eff }}=1.5$ and 1.55 might be somewhat affected by the system size effect. It is worth noting that the Monte Carlo simulation of a dense polymer by Ray and Binder [18 shows that the monomer diffusion constant decreases with increasing the system size. 


\section{SUMMARY AND DISCUSSIONS}

Most of our findings in this work have been obtained from numerical analysis only without first principle derivations. Nevertheless, we believe that they pose new problems and suggest new experiments. We make some discussions mentioning possible experiments below. 1) Introducing the concept of bond breakage, we have succeeded to quantitatively analyze the kinetic heterogeneities in simple model systems, which have been witnessed by a number of the authors. As shown in Fig.6, strings composed of broken bonds are very frequent and they aggregate at low temperatures to form clusters. The bond breakage time $\tau_{b}$ is related to the correlation length $\xi$ as (4.4). In future work we should clarify the relationship of our patterns in the $\alpha$ relaxation and those by Muranaka and Hiwatari [9] on a much shorter time scale.

2) The weakly bonded regions identified by the bond breakage are purely dynamical objects. Large scale heterogeneities have not been clearly detected in snapshots of the usual physical quantities such as the densities, the stress tensor, the kinetic energy (=temperature), etc. On the other hand, in granular matters in shear flow [30, stress heterogeneities have been observed optically by using birefringent materials. We admit the possibility that such stress heterogeneities also exist in supercooled liquids but are masked by the thermal fluctuations. We will check this point in future.

3) It is of great interest how the kinetic heterogeneities, which satisfy the dynamic scaling (4.4), evolve in space and time and why they look so similar to the critical fluctuations in Ising systems in the mean field level. In our steady state problem $T$ and $\dot{\gamma}$ are two relevant scaling fields, the critical point being located at $T=\dot{\gamma}=0$. No divergence has been detected at a nonzero temperature in our simulations.

4) In his experiments Fischer [51] has reported large excess light scattering with a correlation length $\xi(20 \sim 200$ $\mathrm{nm})$ which increases on approaching the glass transition from a liquid state. This indicates the presence of very large scale density heterogeneities in supercooled liquids, which is often called Fischer's clusters. Motivated by this effect, Weber et al [52] performed Monte Carlo simulations on a dense polymer and found that short range nematic orientational order can give rise to enhancement of long range density fluctuations. They expected that such anisotropic interactions could be the origin of Fischer's clusters. This suggests that Fischer's clusters do not exist in liquids composed of structureless particles.

5) We have examined nonlinear rheology in glassy states. The rheological relations obtained are simplest among those consistent with the experiments 24 26. The mechanism of the non-Newtonian behavior in supercooled liquids is conceptually new and should be further examined in experiments such as in colloidal systems in glassy states. In particular, polymers should exhibit pro- nounced non-Newtonian behavior, as the glass transition is approached, even without entanglement. Rheology of chain systems remains totally unexplored near the glass transition.

6) In our systems small anisotropic changes of the pair correlation functions $g_{\alpha \beta}(r)$ near the first peak $\left(\sim \sigma_{\alpha \beta}\right)$ can give rise to the limiting shear stress $\sigma_{\text {lim }}$, which is $3 \sim 5 \%$ of the pressure in our case. Note that our systems are highly compressed with high pressure. However, the pressure needs not be very high in supercooled liquids in the presence of an attractive part of the potential. Even in such cases, we expect that $\sigma_{\text {lim }}$ is a few $\%$ of the shear modulus. This is suggested by the previous work on amorphous alloys 31 35, where the yield stress $\sigma_{y}$ in the inhomogeneous case (in which shear bands appear) is known to be $2 \sim 3 \%$ of the shear modulus.

7) Stillinger expected that in fragile glass-forming liquids shear flow occurs by tear and repair of slipping walls separating strongly bonded regions [7]. We have not observed such localization of slips or jump at least in our temperature range. But there might be a tendency that broken bonds form surfaces at low temperatures in $3 \mathrm{D}$, though not conspicuous, which should be checked in future.

8) There is no tendency of phase separation for the parameters used. However, there are many cases in which the composition fluctuations are enhanced towards the glass transition temperature. It is of great interest how the two transitions influence each other [53,54]. It is also known that shear flow can induce composition fluctuation enhancement in asymmetric viscoelastic mixtures, when emergence of less viscous regions can reduce the effective viscosity 23]. We expect that this effect can come into play also in supercooled liquids, for example, for large enough size ratios or in the presence of small attraction between the two components. Experiments to detect this effect seem to be promising in colloidal systems.

9) We have introduced the time correlation function $F_{s}(q, t)$ in shear and found its simple relaxation behavior in Fig.15. It coincides with the usual time correlation function for $q_{x}=0$ or when the scattering vector is perpendicular to the flow direction. Dynamic scattering experiments in shear flow would be very informative to detect the shear-induced diffusion [23]. A direct diffusion measurement in sheared supercooled fluids is also very interesting, which we will be analyzed in the appendix. Though our system size is still too small, we have detected a tendency of the breakdown of the Einstein-Stokes relation in $3 \mathrm{D}$ to obtain $D \sim \eta^{-\nu}$ with $\nu=0.75 \sim 0.8$.

10) In strong shear the structural relaxation is characterized by $\tau_{\alpha} \sim 0.1 \tau_{b} \sim 0.1 / \dot{\gamma}$ as (6.8). This nonlinear effect could be measured as drastic reduction of the rotational relaxation time by dielectric response or by more sophisicated techniques 36,37 from sheared supercooled liquids. The same effect is expected for periodic shear flow. 
11) Understanding of transient mechanical response in terms of the kinetic heterogeneities is of great importance. For example, we have found a stress overshoot after application of shear strain in accord with the experiments [24,25]. We should also understand glassy behavior of the complex shear modulus against small periodic shear [55]. On these topics we will report shortly. 12) In our systems we have not yet found essential differences between $2 \mathrm{D}$ and $3 \mathrm{D}$ except for the difference in the value of the dynamic exponent $z$ in (4.4). We believe that a large part of essential ingredients of glassy dynamics can be understood even in two dimensions.

13) In a forthcoming paper we will focus our attention on jump motions of particles over distances longer than $\sigma_{1}$. They will be shown to occur heterogeneously in space and determine the diffuison constant. These heterogeneity structures are essentially the same as those in the bond breakage processes studied in this paper.

\section{ACKNOWLEDGMENTS}

We thank Dr. T. Muranaka, Professor Y. Hiwatari, Professor K. Kawasaki, Professor P. Harrowell, Dr. D. Perera, and Professor A.J. Liu for helpful discussions. Thanks are also due to Professor S. Takeuchi, who kindly sent his work on amorphous alloys. This work is supported by Grants in Aid for Scientific Research from the Ministry of Education, Science and Culture. Calculations have been carried out at the Supercomputer Laboratory, Institute for Chemical Research, Kyoto University and the Computer Center of the Institute for Molecular Science, Okazaki, Japan.

\section{APPENDIX}

Let us calculate the variances among the $x, y$, and $z$ components, $x_{j}(t)-x_{j}(0), y_{j}(t)-y_{j}(0)$, and $z_{j}(t)-z_{j}(0)$, of the net displacement vector $\boldsymbol{r}_{j}(t)-\boldsymbol{r}_{j}(0)$ of the $j$ th particle in shear flow. We fix its initial position $\boldsymbol{r}_{j}(0)$ at $\boldsymbol{r}_{0}=\left(x_{0}, y_{0}, z_{0}\right)$. The average displacement arises from the convection as

$$
\left\langle\boldsymbol{r}_{j}(t)-\boldsymbol{r}_{j}(0)\right\rangle=\dot{\gamma} t y_{0} \boldsymbol{e}_{x} .
$$

Assuming the isotropy of the subtracted displacement (6.2), which is suggested by Fig.18, we may write the variances of the $y$ and $z$ components are

$$
G(t)=\left\langle\left(y_{j}(t)-y_{j}(0)\right)^{2}\right\rangle=\left\langle\left(z_{j}(t)-z_{j}(0)\right)^{2}\right\rangle .
$$

The variance of the $x$ component then becomes

$$
\left\langle\left(x_{j}(t)-x_{j}(0)-\dot{\gamma} t y_{0}\right)^{2}\right\rangle=G(t)+2 \dot{\gamma}^{2} \int_{0}^{t} d t_{1}\left(t-t_{1}\right) G\left(t_{1}\right) .
$$

The cross correlation exists between the $x$ and $y$ components as

$$
\left\langle\left(x_{j}(t)-x_{j}(0)\right)\left(y_{j}(t)-y_{j}(0)\right)\right\rangle=\dot{\gamma} \int_{0}^{t} d t_{1} G\left(t_{1}\right) .
$$

In the diffusion time regime $t \gtrsim \tau_{\alpha}$ we may set $G(t)=2 D t$ to obtain

$$
\begin{gathered}
\left\langle\left(x_{j}(t)-x_{j}(0)-\dot{\gamma} t y_{0}\right)^{2}\right\rangle \cong 2 D t\left(1+\frac{1}{3} \dot{\gamma}^{2} t^{2}\right), \\
\left\langle\left(x_{j}(t)-x_{j}(0)\right)\left(y_{j}(t)-y_{j}(0)\right)\right\rangle \cong D \dot{\gamma} t^{2} .
\end{gathered}
$$

Note that $D$ is strongly dependent on $\dot{\gamma}$ in strong shear as shown in Fig.19. Measurements of the above variances are very informative.
[1] J. Jäckle, Rep. Prog. Phys., 49171 (1986).

[2] M.D. Ediger, C.A. Angell and S.R. Nagel, J. Phys. Chem. 100, 13200 (1996).

[3] U. Bengtzelius, W. Götze and A. Sjölander, J. Phys. C 17, 5915 (1984).

[4] E. Leutheusser: Phys. Rev. A 29, 2765 (1984).

[5] G. Adam and J.H. Gibbs, J. Chem. Phys. 43, 139 (1965).

[6] M.H. Cohen and G.S. Grest, Phys. Rev. B 20, 1077 (1979).

[7] F.H. Stillinger, J. Chem. Phys. 89, 6461 (1988).

[8] K.L. Ngai, R.W. Rendell and D.J. Plazek, J. Chem. Phys. 94, 3018 (1991).

[9] T. Muranaka and Y. Hiwatari, Phys. Rev. E 51, R2735 (1995).

[10] T. Muranaka and Y. Hiwatari, Supplement to Prog. Theor. Phys. 126, 403 (1997).

[11] M.M. Hurley and P. Harrowell, Phys. Rev. E 52, 1694 (1995).

[12] D.N. Perera and P. Harrowell, Phys. Rev. E 54, 1652 (1996) ; J. Non-Cryst. Solids, the proceedings for the Vigo meeting (Vigo, June 30- July 11, 1997);

[13] R. Yamamoto and A. Onuki, J. Phys. Soc. Jpn., 662545 (1997).

[14] R. Yamamoto and A. Onuki, Europhys. Lett. 40, 61 (1997).

[15] A. Onuki and R. Yamamoto, J. Non-Cryst. Solids, the proceedings for the Vigo meeting (Vigo, June 30- July 11, 1997); the proceedings for the Towa conference (Fukuoka, Nov. 4-7, 1997).

[16] W. Kob, C. Donati, S.J. Plimton, P.H. Poole, and S.C. Glotzer, Phys. Rev. Lett. 79, 2827 (1997).

[17] C. Donati, J.F. Douglas, W. Kob, S.J. Plimton, P.H. Poole, and S.C. Glotzer, preprint.

[18] P. Ray and K. Binder, Europhys. Lett. 2753 (1994).

[19] C. Dasgupta, A.V. Indrani, S. Ramaswamy and M.K. Phani, Europhys. Lett. 15, 307 (1991). 
[20] R.M. Ernst, S.R. Nagel and G.S. Grest, Phys. Rev. B 43, 8070 (1991).

[21] S.S. Ghosh and C. Dasgupta, Phys. Rev. Lett. 77, 1310 (1996).

[22] D.J. Salvino, S. Rogge, B. Tigner and D.D. Osheroff, Phys. Rev. Lett. 73, 268 (1994) ; S. Rogge, D. Natelson and D.D. Osheroff, J. Low Temp. Phys. 106, 767 (1997).

[23] A. Onuki, J. Phys. C 9, 6119 (1997).

[24] J.H. Simmons, R.K. Mohr and C.J. Montrose, J. Appl. Phys. 53, 4075 (1982).

[25] J.H. Simmons, R. Ochoa, K.D. Simmons and J.J. Mills, J. Non-Cryst. Solids 105, 313 (1988).

[26] Y. Yue and R. Brückner, J. Non-Cryst. Solids, 18066 (1994).

[27] T. Okuzono and K. Kawasaki, Phys. Rev. E, 51, 1246 (1995).

[28] D.J. Durian, Phys. Rev. E, 55, 1739 (1997).

[29] S.A. Langer and A.J. Liu, J. Phys. Chem. B 101, 8667 (1997).

[30] B. Miller, C.O'Hern and R.P. Behringer, Phys. Rev. Lett. 77, 3110 (1996) ; R. Khosropour, J. Zirinsky, H.K. Pak and R.P. Behringer, Phys. Rev. E, 56, 4467 (1997).

[31] H.S. Chen and M. Goldstein, J. Appl. Phys. 43, 1642 (1971).

[32] F. Spaepen, Acta Metall. 25, 407 (1977).

[33] A.S. Aragon, Acta Metall. 27,47 (1979).

[34] K. Maeda and S. Takeuchi, Phys. Stat. Sol. 49, 685 (1978) ; S. Kobayashi, K. K. Maeda and S. Takeuchi, Acta Metall. 28 , 1641 (1980); K. Maeda and S. Takeuchi, Phil. Mag. A 44, 643 (1981).

[35] S. Takeuchi and K. Maeda, in Metallic and Semiconducting Glasses, ed. A. Bhatnagar, (Trans. Tech. Pub., 1987) and references quoted therein.

[36] F. Fujara, B. Geil, H. Silescu and G. Fleischer, Z. Phys. B 88, 195 (1992); I. Chang, F. Fujara, B. Geil, G. Heuberger, T. Mangel and H. Silescu, J. Non-Cryst. Solids 172-174, 248 (1994).

[37] M.T. Cicerone, F.R. Blackburn and M.D. Ediger, Macromolecules 28, 8224 (1995); M.T. Cicerone and M.D. Ediger, J. Chem. Phys. 104, 7210 (1996).

[38] F.H. Stillinger and A. Hodgdon, Phys. Rev. E, 50, 2064 (1994).

[39] D. Richter, R. Frick and B. Farago, Phys. Rev. Lett. 61, 2465 (1988).

[40] D. Thirumalai and R.D. Mountain, Phys. Rev. E 47, 479 (1993).

[41] D. Perera and P. Harrowell, private communication. They have also reproduced the dynamical scaling exponent $z \cong 4$ in a $2 \mathrm{D}$ simulation using a different definition of $\xi$.

[42] J. Matsui, T. Odagaki and Y. Hiwatari, Phys. Rev. Lett. 73, 2452 (1994).

[43] B. Bernu, Y. Hiwatari and J.P. Hansen, J. Phys. C 18, L371 (1985); B. Bernu, J.P. Hansen, Y. Hiwatari and G. Pastore Phys. Rev. A 36, 4891 (1987).

[44] D. Henderson and P.J. Lenard, Physical Chemistry, an Advanced Treatise, ed. D. Henderson (Academic, 1971) p. 414.

[45] J.P. Hansen and I.R. McDonald, Theory of Simple Liquids (Academic, 1986).

[46] M.P. Allen and D.J. Tildesley, Computer Simulation of
Liquids, (Clarendon, Oxford, 1987).

[47] D.J. Evans and G.P. Morriss, Statistical Mechanics of Nonequilibrium Liquids (Academic, New York, 1990).

[48] J.G. Kirkwood and F.P. Buff, J. Chem. Phys. 19, 774 (1951).

[49] H.J.M. Hanley, D.J. Evans, and S. Hess, J. Chem. Phys., 781440 (1983).

[50] A. Onuki and K. Kawasaki, Phys. Lett. A 72, 233 (1979); B.J. Ackerson and N.A. Clark, J. Physique 42, 929 (1981).

[51] E.W. Fischer, Physica A 201, 183 (1993).

[52] H. Weber, W. Paul, W. Kob and K. Binder, Phys. Rev. Lett. 78, 2136 (1997).

[53] A. Sappelt and J. Jäckle, Physica A, 240, 453 (1997).

[54] G. Meier, D. Vlassopoulos and G. Fytas Europhys. Lett. 30, 325 (1995).

[55] N. Menon, S.R. Nagel and D.C. Venerus Phys. Rev. Lett., 73, 963 (1994). 


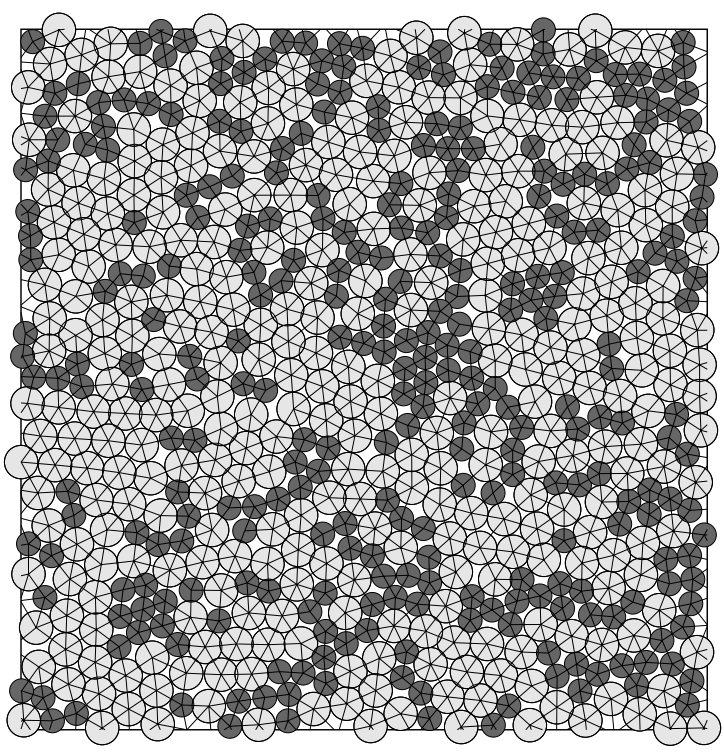

FIG. 1. A typical particle configuration and the bonds defined at a given time at $\Gamma_{\text {eff }}=1.4$ in $2 \mathrm{D}$. The diameters of the circles here are equal to $\sigma_{\alpha}$. The areal fraction of the soft-core regions is $93 \%$. A $1 / 16$ of the total system is shown.
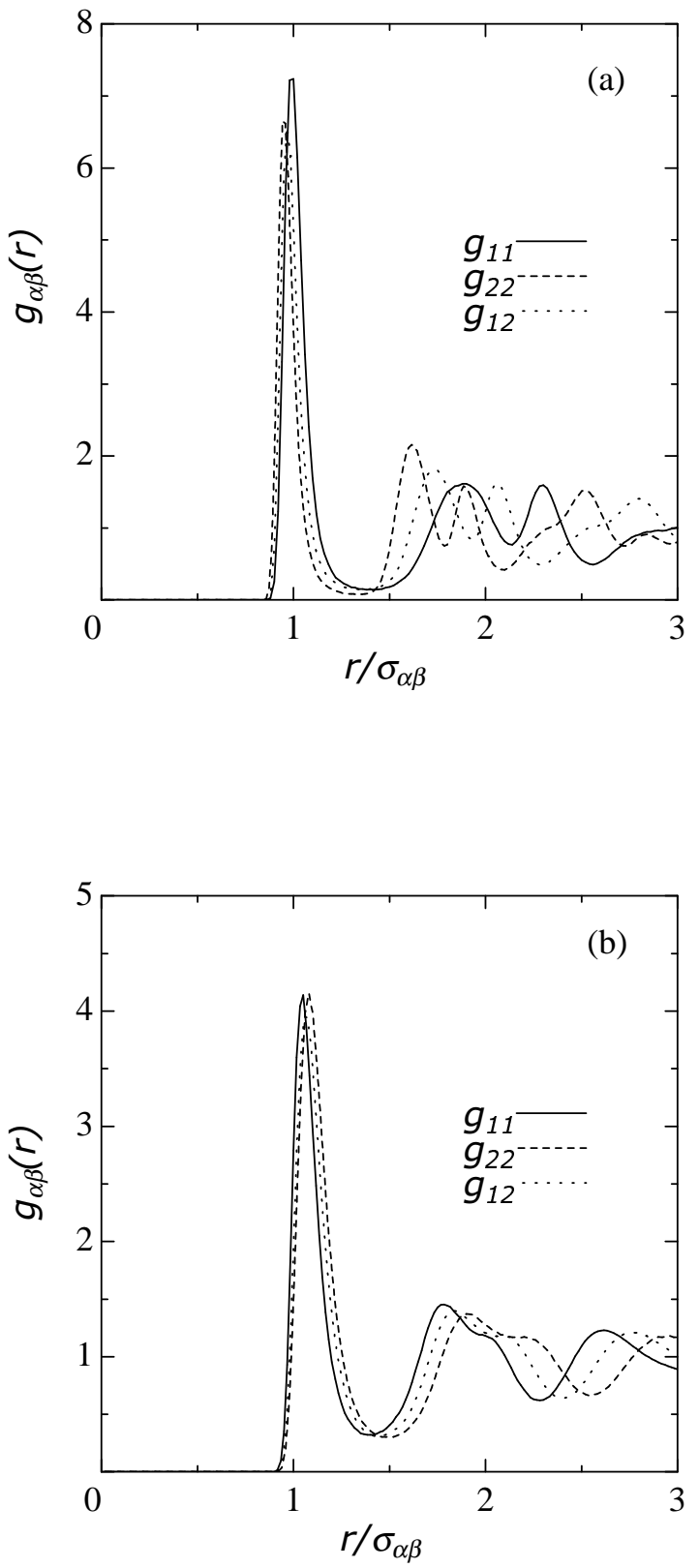

FIG. 2. The pair correlation functions $g_{\alpha \beta}(r)$ in quiescent states as functions of $r / \sigma_{\alpha \beta}$ at $\Gamma_{e f f}=1.4$ in $2 \mathrm{D}$ (a) and at $\Gamma_{\text {eff }}=1.55$ in $3 \mathrm{D}(\mathrm{b})$. 

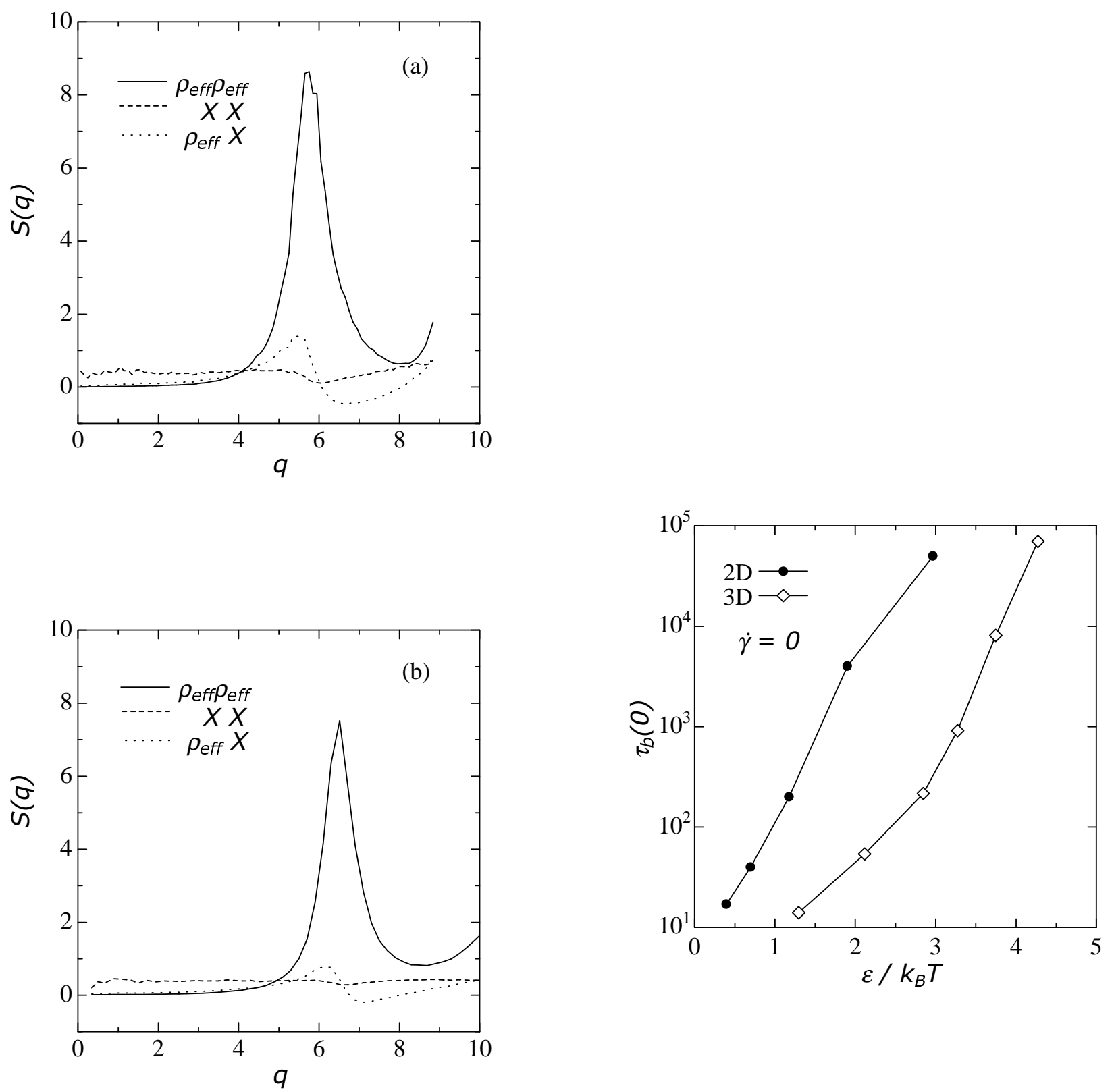

FIG. 3. The structure factors $S(q)$ defined in (3.3)- (3.5) in quiescent states at $\Gamma_{\text {eff }}=1.4$ in $2 \mathrm{D}$ (a) and at $\Gamma_{\text {eff }}=1.55$ in $3 \mathrm{D}$ (b). The dimensionless wavenumber $q$ is measured in units of $\sigma_{1}^{-1}$. The solid, dashed, and dotted lines correspond to $\rho_{e f f}-\rho_{e f f}, X-X$, and $\rho_{e f f}-X$ correlations, respectively.

FIG. 4. Temperature dependence of the bond breakage time $\tau_{b}(0)$ at zero shear $(\bullet)$ in $2 \mathrm{D}$ and $(\diamond)$ in $3 \mathrm{D}$. The $\epsilon$ is the potential parameter in the soft-core potentials (2.1). The time is measured in units of $\tau_{0}$ in $(2.2)$, so $\tau_{b}(0)$ is dimensionless. 

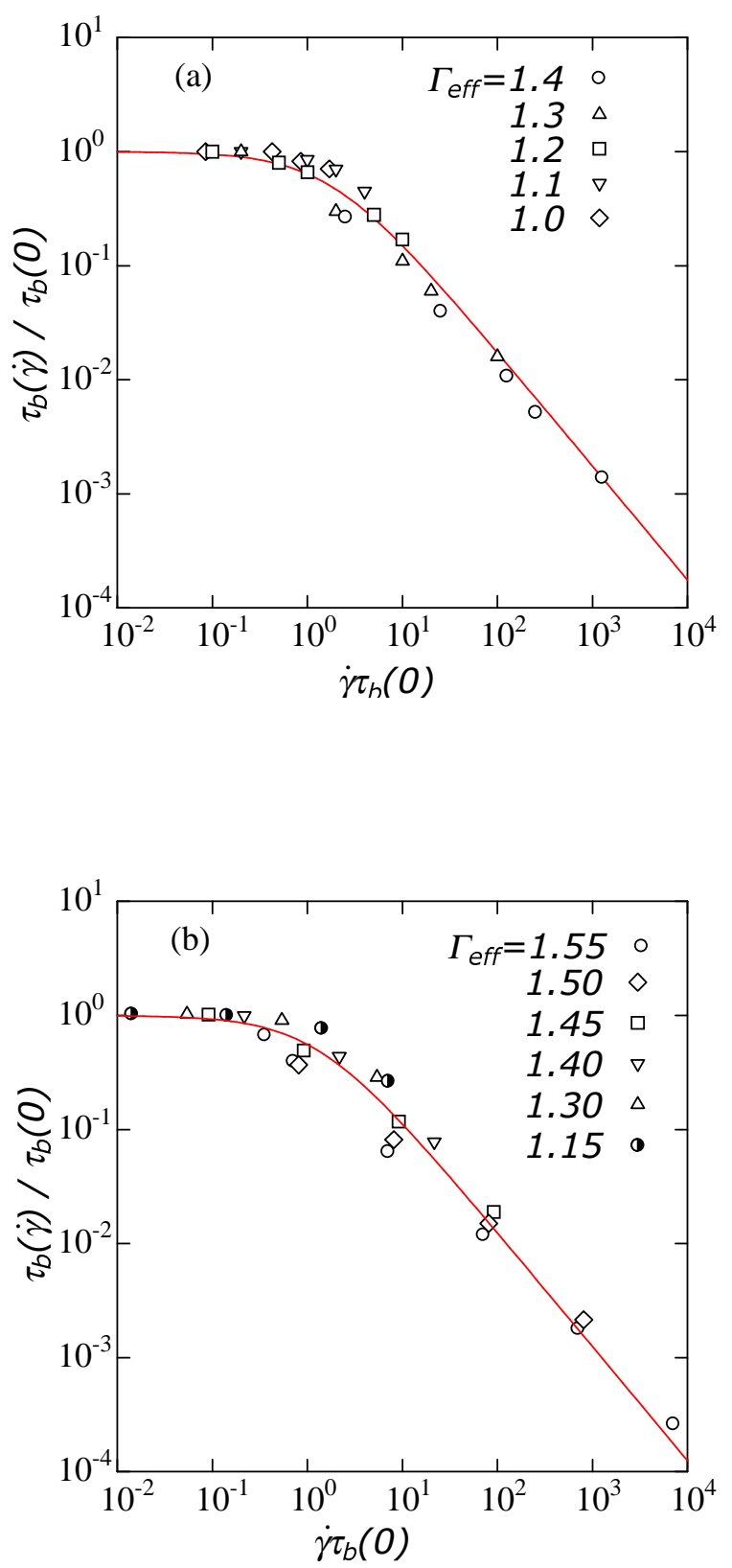

FIG. 5. The normalized bond breakage time $\tau_{b}(\dot{\gamma}) / \tau_{b}(0)$ versus $\dot{\gamma} \tau_{b}(0)$ for various $\Gamma_{\text {eff }}$ in $2 \mathrm{D}$ (a) and $3 \mathrm{D}$ (b). All the data collapse on the curve $1 /\left(1+A_{b} x\right)$ with $x=\dot{\gamma} \tau_{b}(0)$.

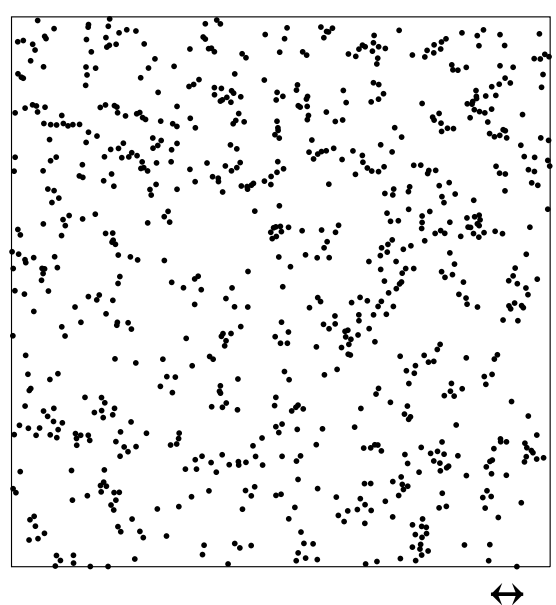

(a) $\Gamma_{\text {eff }}=1, \dot{\gamma}=0$

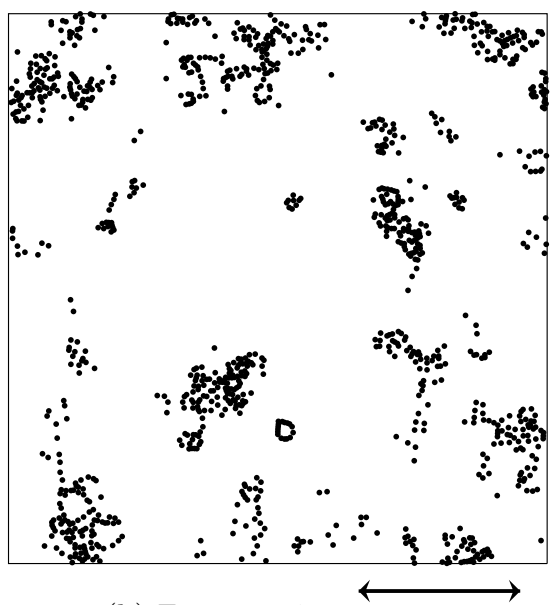

(b) $\Gamma_{\text {eff }}=1.4, \dot{\gamma} \stackrel{\longleftrightarrow}{=}$

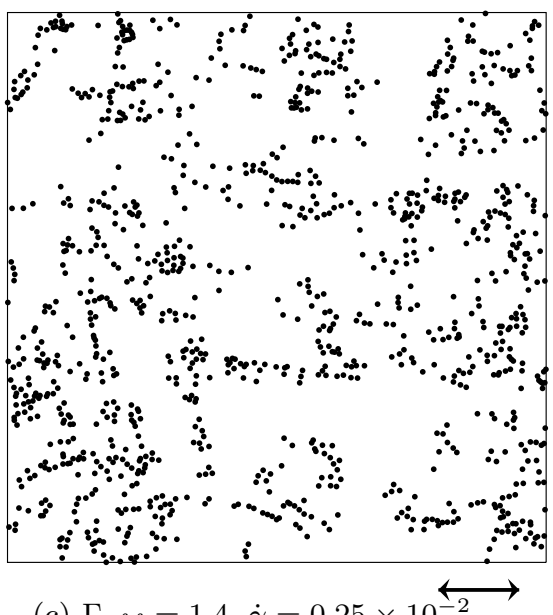

(c) $\Gamma_{\text {eff }}=1.4, \dot{\gamma}=0.25 \times 10^{\overleftarrow{-2}}$

FIG. 6. Snapshots of the broken bonds in 2D without shear. The system length is $118 \sigma_{1}$. Here $\Gamma_{\text {eff }}=1$ with weak heterogeneity (a), and $\Gamma_{\text {eff }}=1.4$ with enhanced heterogeneity (b). For $\dot{\gamma}=2.5 \times 10^{-2}$ (c), the heterogeneity is much suppressed. The flow is in the upward direction and the velocity gradient is in the horizontal direction from left to right. The arrows indicate the correlation length $\xi$ obtained from (4.2). 


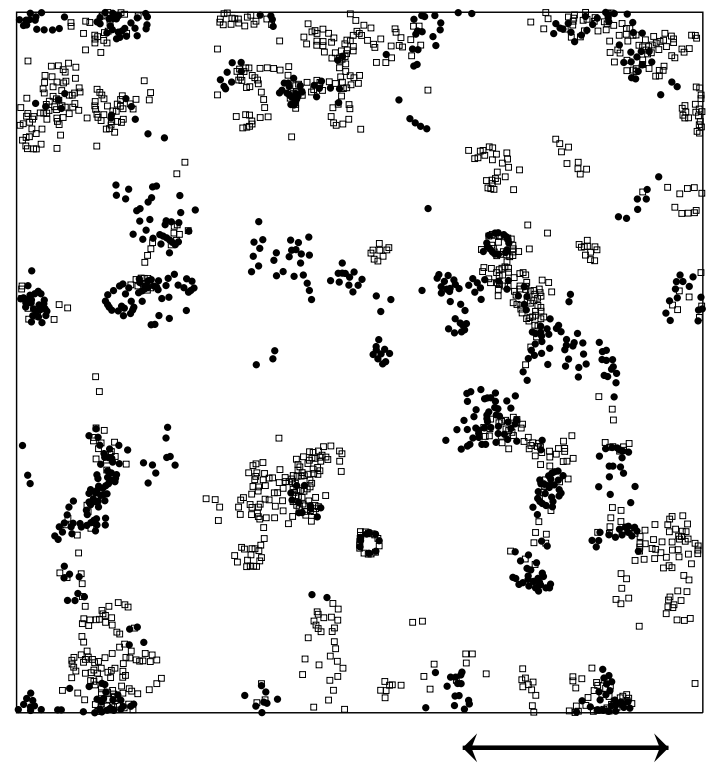

FIG. 7. Broken bond distributions in two consecutive time intervals, $\left[t_{0}, t_{0}+0.05 \tau_{b}\right](\square)$ and $\left[t_{0}+0.05 \tau_{b}, t_{0}+0.1 \tau_{b}\right](\bullet)$, at $\Gamma_{\text {eff }}=1.4$ in $2 \mathrm{D}$. The arrow indicates $\xi$.
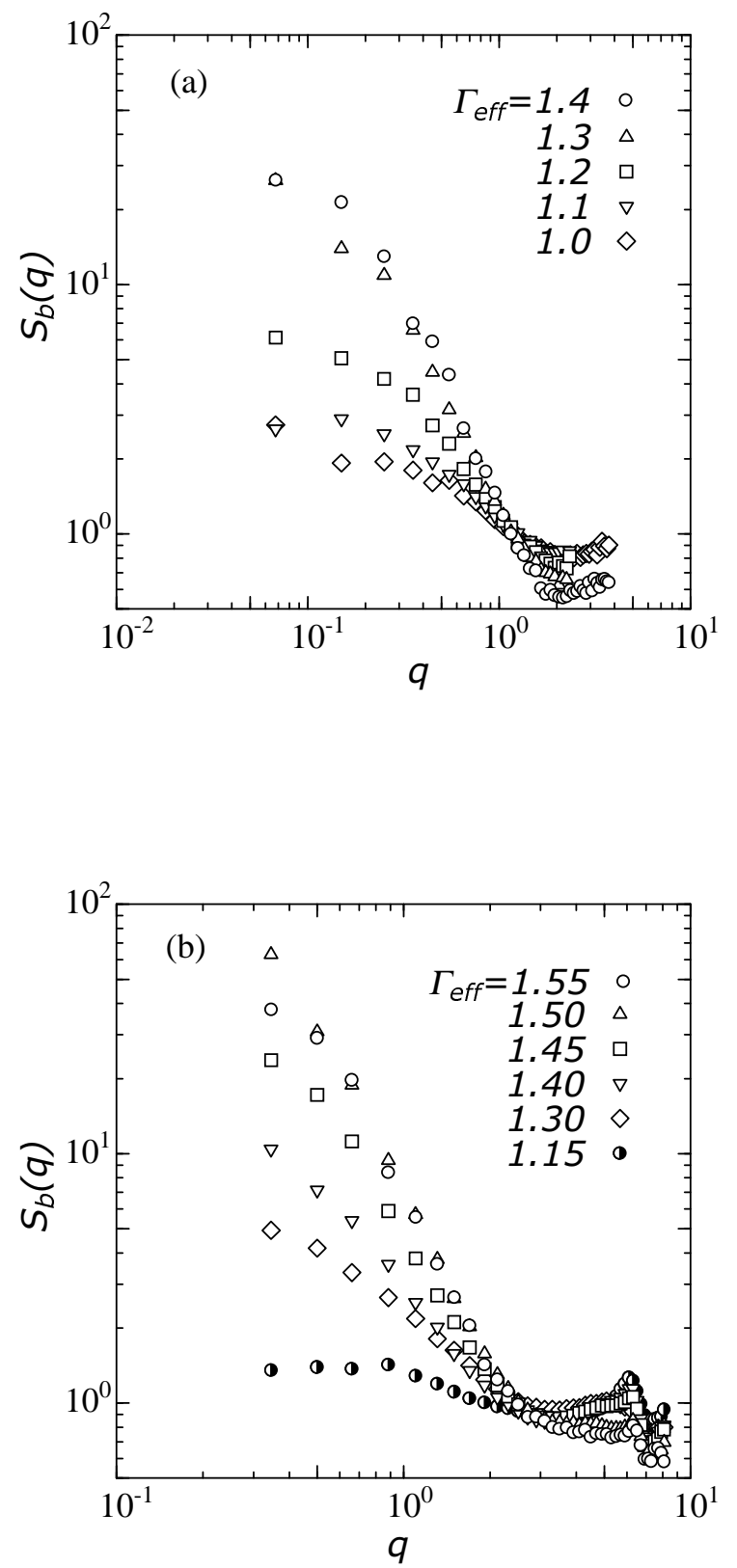

FIG. 8. $S_{b}(q)$ versus $q$ on logarithmic scales for various $\Gamma_{\text {eff }}$ at $\dot{\gamma}=0$ in 2D (a) and 3D (b). Its long wavelength limit is of order $\xi^{2}$ as (4.3). 

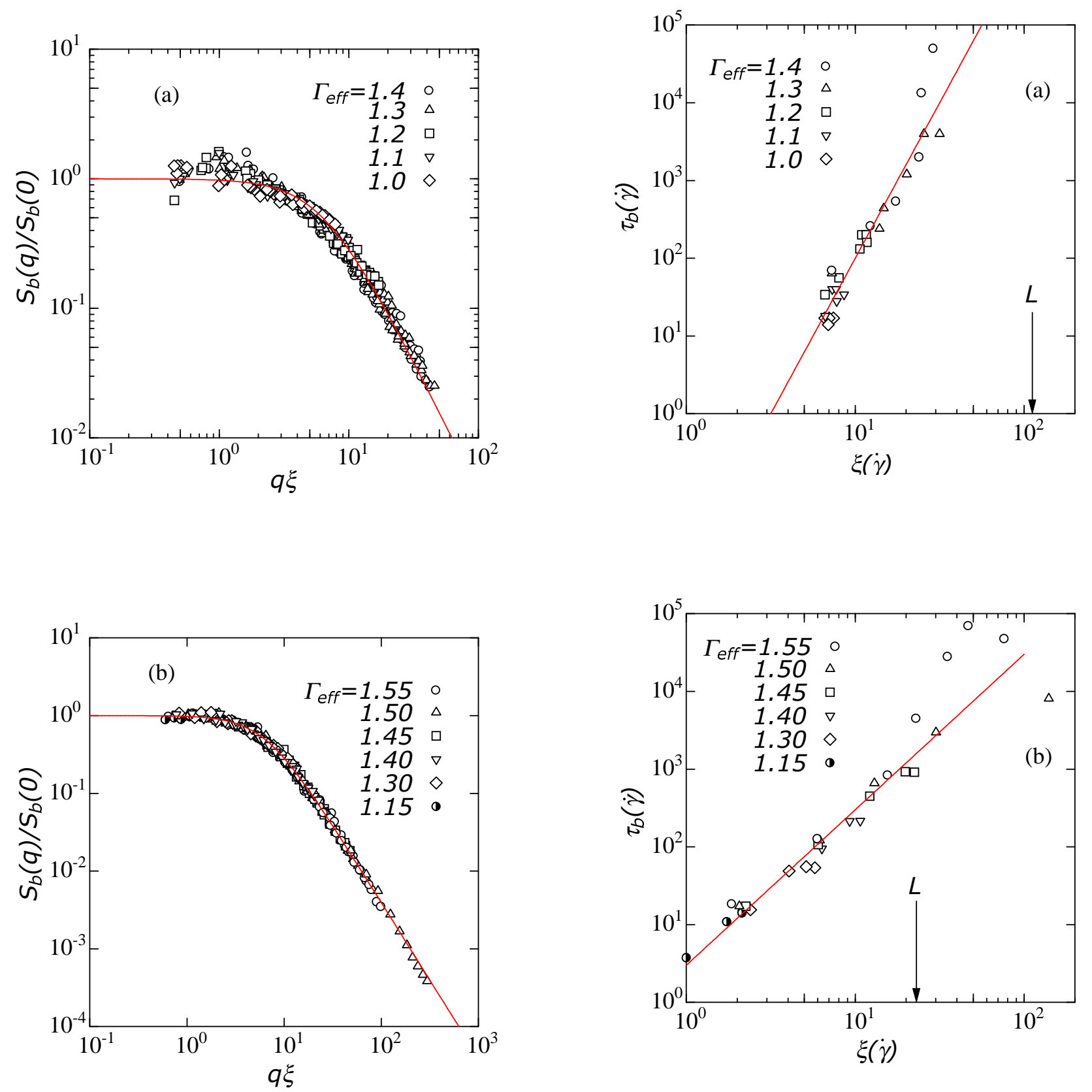

FIG. 9. $S_{b}(q) / S_{b}(0)$ on logarithmic scales for various $\Gamma_{e f f}$ and $\dot{\gamma}$ in 2D (a) and 3D (b). The solid line is the Ornstein-Zernike form $1 /\left(1+x^{2}\right)$ with $x=q \xi$. 

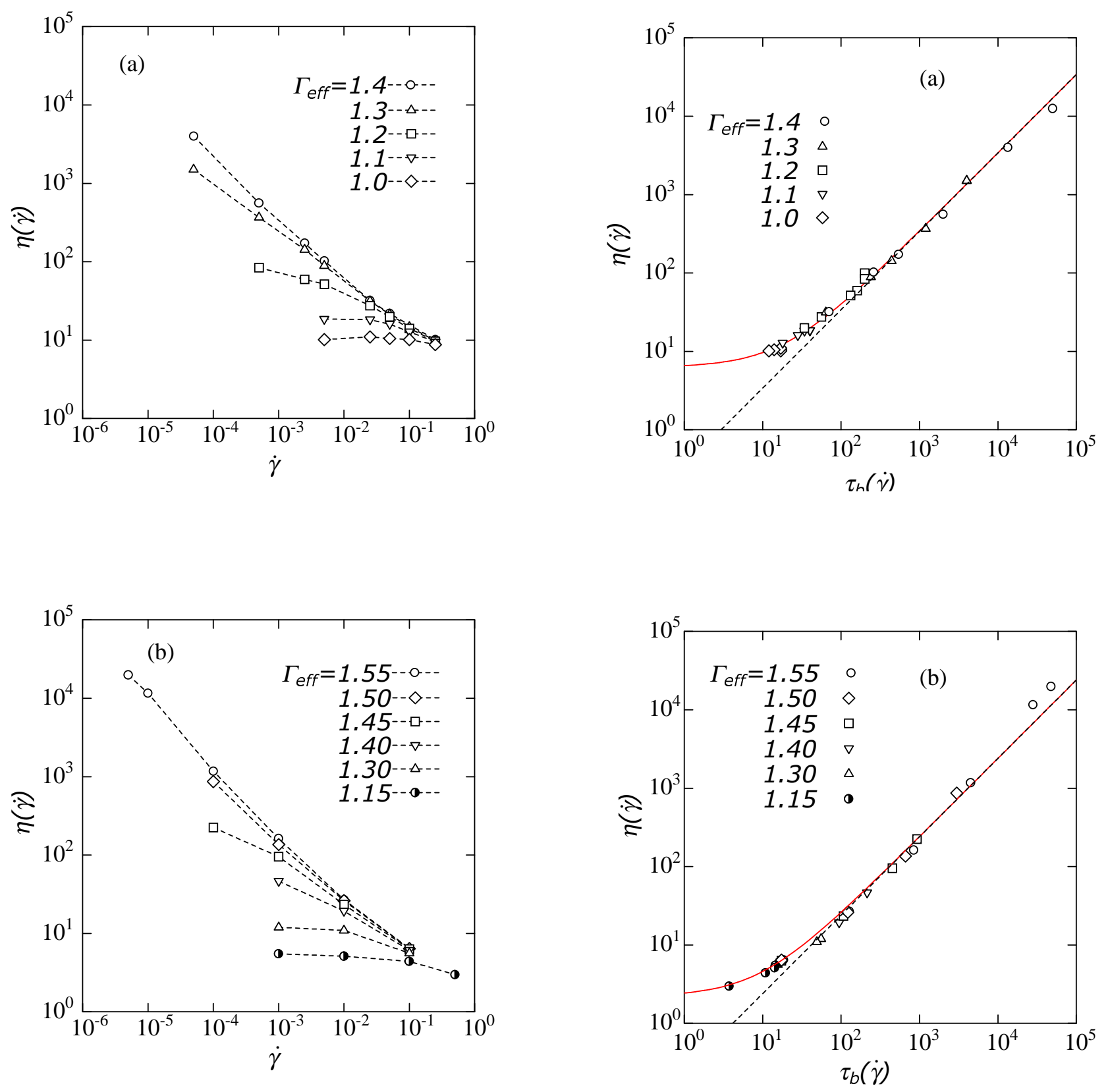

FIG. 11. The viscosity $\eta(\dot{\gamma})$ in units of $\epsilon \tau_{0} / \sigma_{1}^{d}$ vesus the shear rate $\dot{\gamma}$ in units of $1 / \tau_{0}$ at various $\Gamma_{\text {eff }}$ in $2 \mathrm{D}$ (a) and $3 \mathrm{D}$ (b). The data tend to become independent of $\Gamma_{\text {eff }}$ at high shear.

FIG. 12. $\eta(\dot{\gamma})$ vesus s $\tau_{b}(\dot{\gamma})$ for various $\Gamma_{\text {eff }}$ in $2 \mathrm{D}(\mathrm{a})$ and 3D (b). The $\eta(\dot{\gamma})$ is determined by $\tau_{b}(\dot{\gamma})$ only irrespectively of $\Gamma_{e f f}$. 

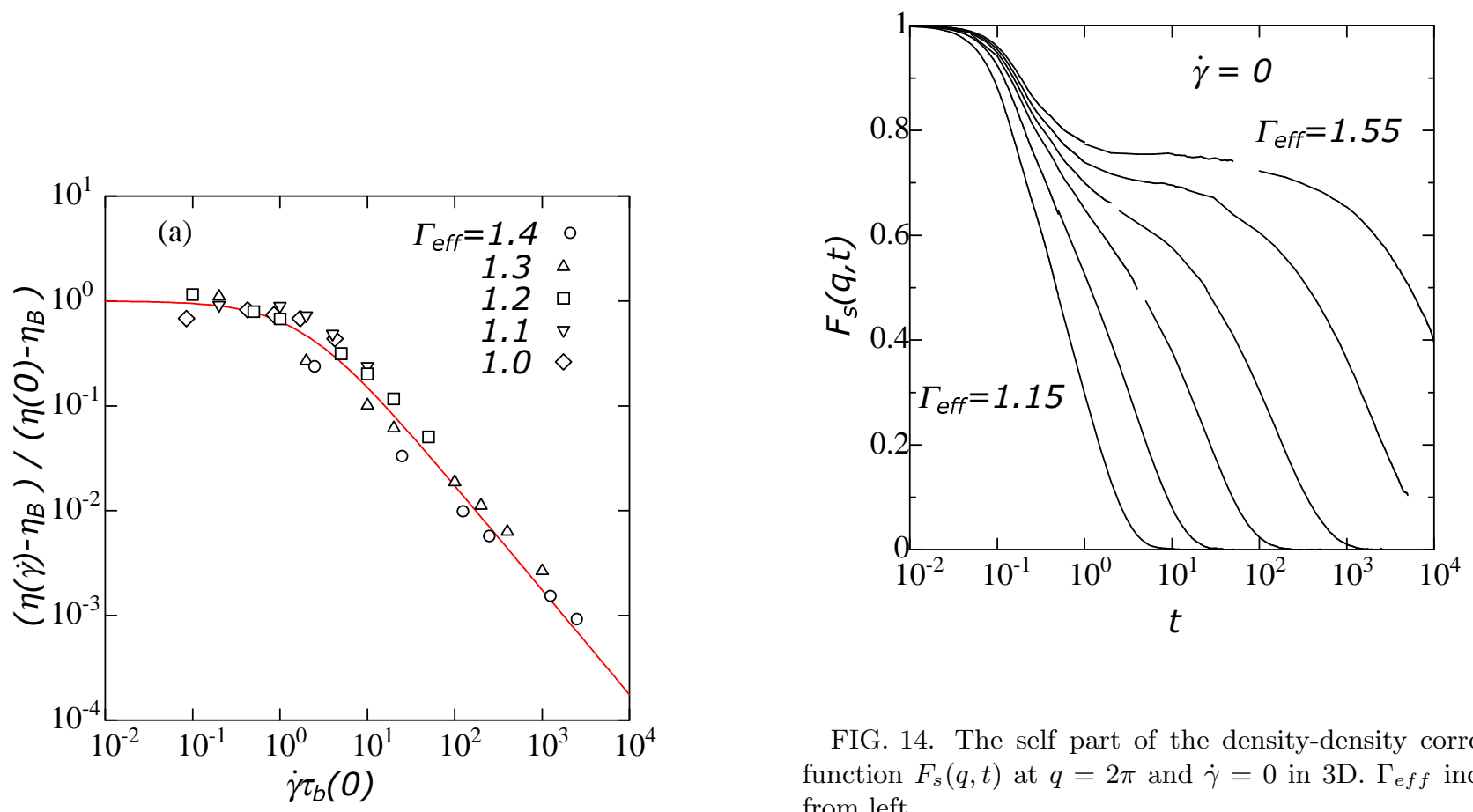

FIG. 14. The self part of the density-density correlation function $F_{s}(q, t)$ at $q=2 \pi$ and $\dot{\gamma}=0$ in $3 \mathrm{D}$. $\Gamma_{\text {eff }}$ increases from left.
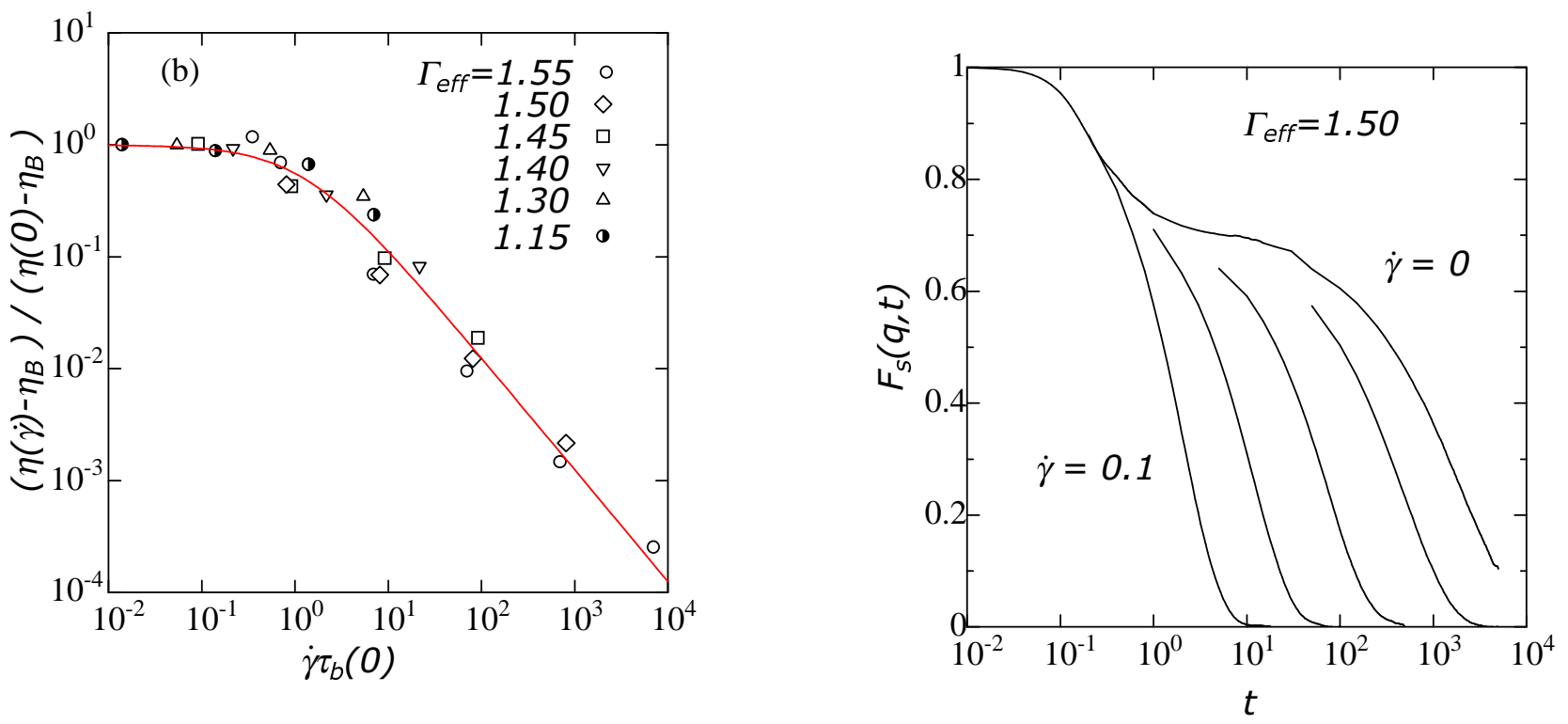

FIG. 15. The time correlation function $F_{s}(q, t)$ at $q=2 \pi$ defined by (6.1) and (6.2) in shear flow, where $\dot{\gamma}=0,10^{-4}$, $10^{-3}, 10^{-2}, 10^{-1}$ from right. The temperature is fixed at

FIG. 13. $\left(\eta(\dot{\gamma})-\eta_{B}\right) /\left(\eta(0)-\eta_{B}\right)$ vs $\dot{\gamma} \tau_{b}(0)$ in $2 \mathrm{D}$ (a) and $3 \mathrm{D}$ (b). The solid curve is $1 /\left(1+A_{b} x\right)$ with $x=\dot{\gamma} \tau_{b}(0)$.

$k_{b} T / \epsilon=0.267\left(\Gamma_{\text {eff }}=1.5\right)$. Increasing $\dot{\gamma}$ is equivalent to raising $T$. 


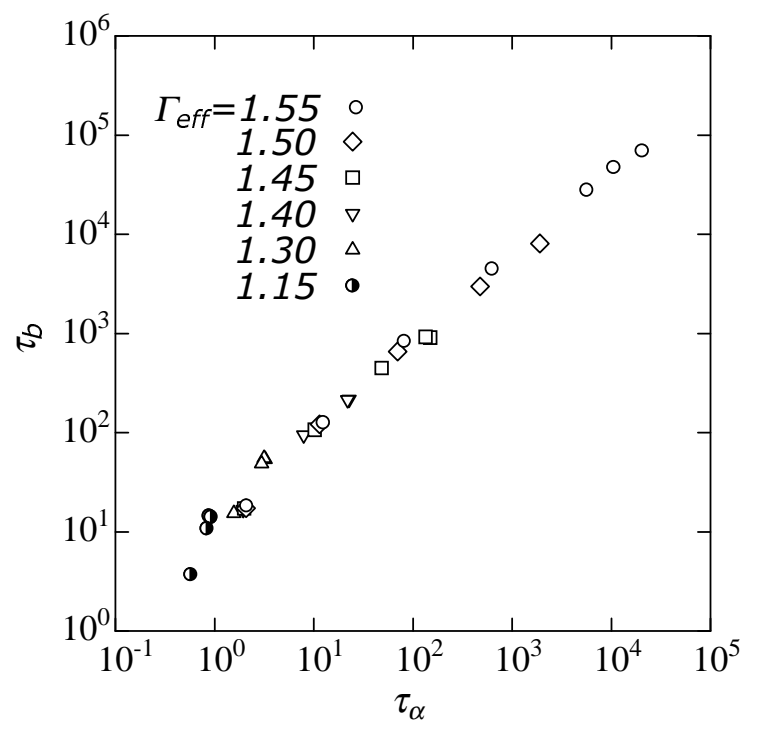

FIG. 16. The linear relationship between $\tau_{\alpha}$ and $\tau_{b}$ for various $\Gamma_{\text {eff }}$ and $\dot{\gamma}$ in 3D. The $\tau_{b}$ is determined from the bond breakage (3.14), and $\tau_{\alpha}$ from the decay of the time correlation function (6.7).

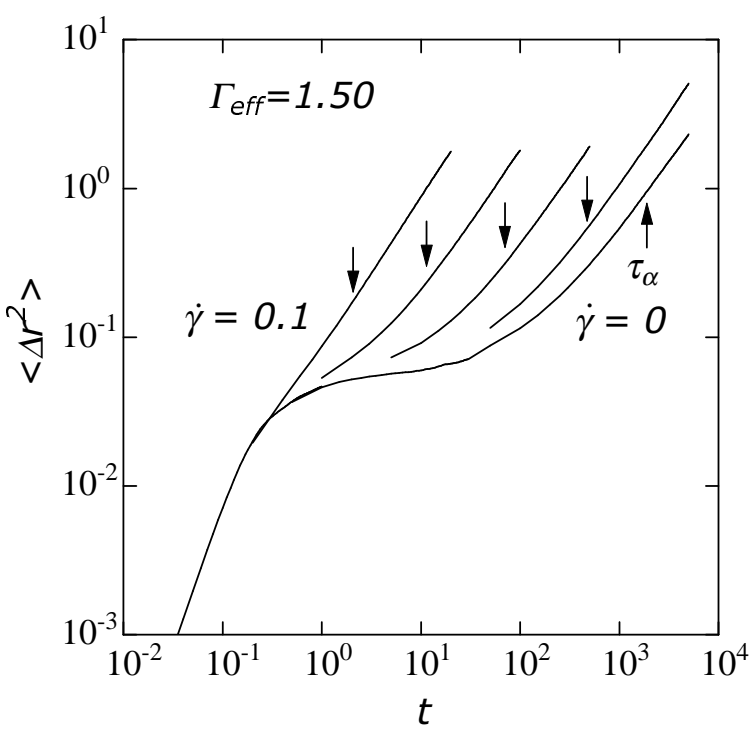

FIG. 17. The mean square displacement in sheared states at $\Gamma_{\text {eff }}=1.5$. The shear rate $\dot{\gamma}$ is $0,10^{-4}, 10^{-3}, 10^{-2}, 10^{-1}$ from right. Increasing $\dot{\gamma}$ is equivalent to raising $T$. The arrows indicate $\tau_{\alpha}$ for each $\dot{\gamma}$. The diffusion (linear) behavior is attained at $t \sim \tau_{\alpha}$.

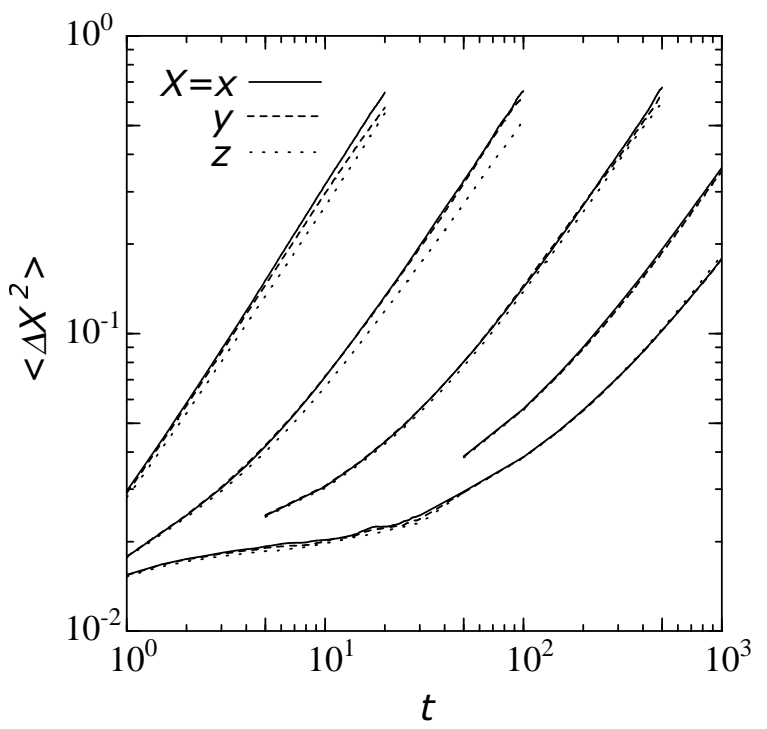

FIG. 18. The mean square displacements of the $x, y$, and $z$ components. They are very close to one another even even in strong shear $\dot{\gamma} \tau_{b}(0) \gg 1$. This demonstrates surprising isotropy of the distribution of the displacement vector (6.2).

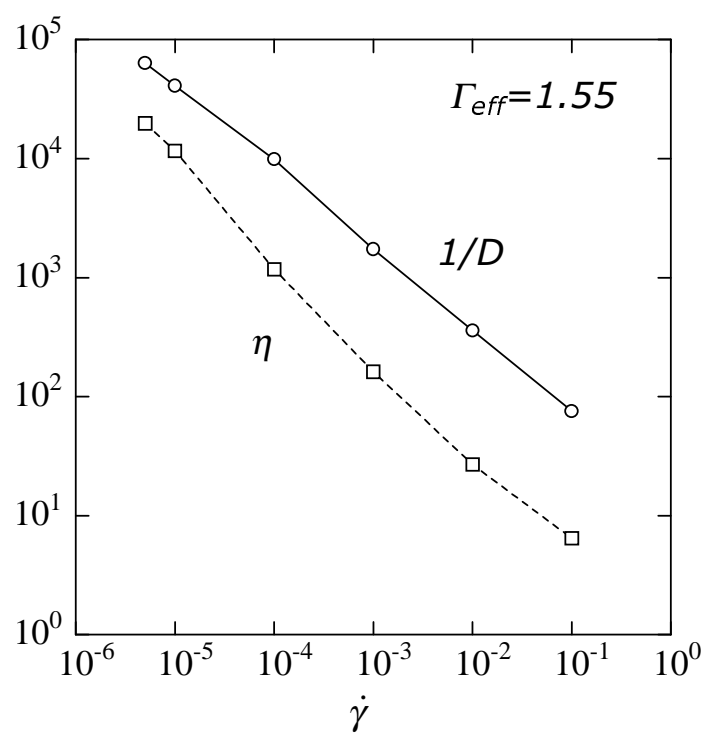

FIG. 19. Shear rate dependences of the inverse diffusion constant $D$ and the viscosity $\eta$ at the lowest temperature, $\Gamma_{\text {eff }}=1.55$. The slope of $D^{-1}$ is noticeably smaller than that of $\eta$. 


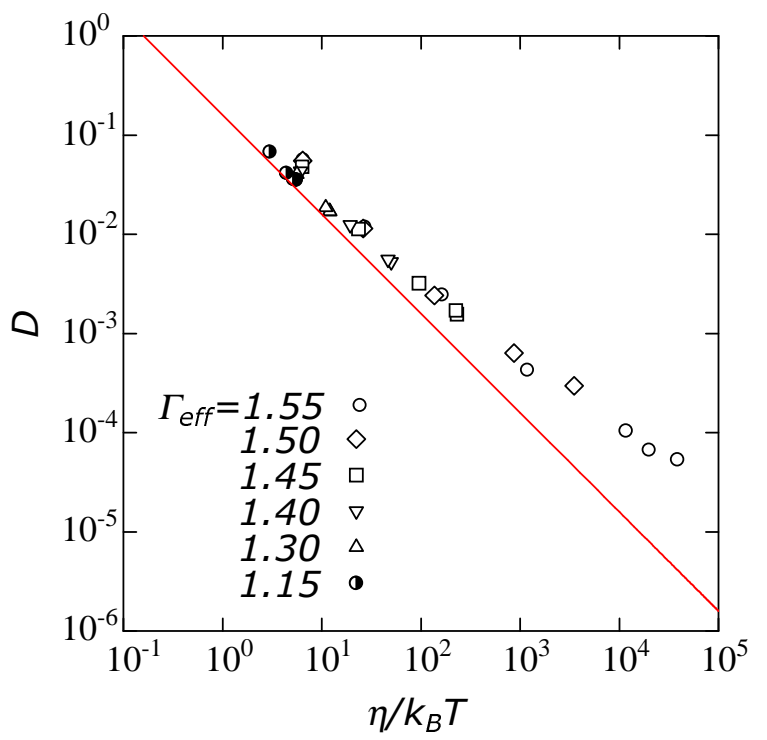

FIG. 20. The diffusion constant $D$ versus the viscosity $\eta$ divided by $k_{B} T$ for various $\Gamma_{\text {eff }}$ and $\dot{\gamma}$. Here $D$ is measured in units of $\sigma_{1}^{2} \tau_{0}^{-1}$ and $\eta / k_{B} T$ in units of $\sigma_{1}^{-d} \tau_{0}$. The solid line represents the Einstein-Stokes formula $D=k_{B} T / 2 \pi \eta \sigma_{1}$,

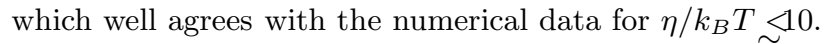

